Special Issue of the Journal of Geodynamics to be published 2002

\title{
Charge generation and propagation in igneous rocks
}

\author{
Friedemann Freund
}

\author{
Department of Physics, San Jose State University, San Jose, California 95192-0106 \\ and SETI Institute/NASA Ames Research Center, MS 239-15 Moffett Field, California 94035-1000, USA \\ Tel: +1-650-604-5183; Fax: +1-650-604-1092; e-mail: ffreund@mail.arc.nasa.gov
}

\section{Abstract}

Various electrical phenomena have been reported prior to or concurrent with earthquakes such as resistivity changes, ground potentials, electromagnetic (EM), and luminous signals. Doubts have been raised as to whether some of these phenomena are real and indeed precursory. One of the reasons for uncertainty is that, despite of decades of intense work, there is still no physically coherent model. Using low-to medium-velocity impacts to measure electrical signals with microsecond time resolution, it has now been observed that when dry gabbro and diorite cores are impacted at relatively low velocities, $\sim 100 \mathrm{~m} / \mathrm{s}$, highly mobile charge carriers are generated in a small volume near the impact point. They spread through the rocks, causing electric potentials exceeding $+400 \mathrm{mV}, \mathrm{EM}$, and light emission. As the charge cloud spreads, the rock becomes momentarily conductive. When a dry granite block is impacted at higher velocity, $\sim 1.5 \mathrm{~km} / \mathrm{s}$, the propagation of the $P$ and $S$ waves is registered through the transient piezoelectric response of quartz. After the sound waves have passed, the surface of the granite block becomes positively charged, suggesting the same charge carriers as observed during the low-velocity impact experiments, expanding from within the bulk. During the next 2-3 ms the surface potential oscillates, indicating pulses of electrons injected from ground and contact electrodes. The observations are consistent with positive holes, e.g., defect electrons in the $\mathrm{O}^{2-}$ sublattice, traveling via the $\mathrm{O} 2 \mathrm{p}$-dominated valence band of the silicate minerals. Before activation, the positive holes lay dormant in the form of electrically inactive positive hole pairs, PHP, chemically equivalent to peroxy links, $\mathrm{O}_{3} \mathrm{X} / \mathrm{OO}_{\mathrm{XO}}$, with $\mathrm{X}=\mathrm{Si}^{4+}, \mathrm{Al}^{3+}$, etc. PHPs are introduced into the minerals by way of hydroxyl, $\mathrm{O}_{3} \mathrm{X}-\mathrm{OH}$, which all nominally anhydrous minerals incorporate when crystallizing in $\mathrm{H}_{2} \mathrm{O}$ laden environments. The fact that positive holes can be activated by low-energy impacts, and their attendant sound waves, suggests that they can also be activated by microfracturing. Depending on where in the stressed rock volume the charge carriers are activated, they will form rapidly moving or fluctuating charge clouds that may account for earthquake-related electrical signals and EM emission. Wherever such charge clouds intersect the surface, high fields are expected, causing electric discharges and earthquake lights.

\section{Introduction}

To more fully understand the earthquake process and allow for the inclusion of electric signals it is necessary to re-evaluate the standard earthquake model as it has evolved historically (Bolt, 1988; Eiby, 1980; Meyer, 1977; Rikitake, 1976). According to the standard model, as rocks in earthquake-prone regions in the Earth's crust are subjected to tectonic stress, they pass through several stages that manifest themselves in ways that lead to measurable effects at the surface of the Earth.

In simple terms these effects can be described as follows: When a rock volume under lithostatic load is compressed, the rocks begin to buckle and undergo microfracturing, causing uplift and tilt at the Earth's surface. The microfracturing releases radon and other gases. Within the crust the electrical conductivity decreases because of the closure of existing pores, then increases as new microcracks are created or saline pore fluids are forced out to form interconnected intergranular water films. As the 
stresses continue to build, larger fissures are thought to allow the intergranular brines to coalesce and further increase the electrical conductivity.

Interestingly, within the framework of this standard model, the rocks themselves play only a passive role. Any change in their electrical conductivity is attributed to the saline pore fluids with which they are saturated and which redistribute in response to the externally applied stress - from closed pores to intergranular brine films to brine-filled fissures. Such a redistribution of fluids, if it occurs, would explain the reported changes in overall electrical conductivity, derived from field measurements. However, a redistribution of fluids can hardly explain the emission of electrical and electromagnetic signals from a given rock volume, except by assuming that, as the brines are forced to flow through narrow fissures and pores, they give rise to streaming potentials (Bernabé, 1998; Draganov et al., 1991; Morrison et al., 1989).

The standard model accords importance to water as the prime cause of electrical conductivity of rocks because crustal earthquakes occur along faults zones. Each fault contains highly deformed, fractured, mellinitized rocks that allow penetration of surface water to $10 \mathrm{~km}$ or more (Park, 1997a,b). Since the conductivity of even moderately saline water far exceeds the conductivity of solid rocks, the average conductivity of a rock volume that includes a fault zone is likely to be dominated by the conductivity of the narrow zones of brine-impregnated faults.

At the same time, faults are bounded on either side by large volumes of rocks that often may not contain much water either in closed pores, as intergranular films or in open fissures. The question arises how the rock volume outside the narrow fault zones contributes to the overall electrical conductivity and specifically to the generation of electrical signals.

This paper presents evidence that dry rocks, in particular dry igneous rocks, can become a source of highly mobile electronic charge carriers. These charge carriers not only cause an increase in the electrical conductivity, but they also appear to be capable of propagating through the rocks as a charge cloud.

\subsection{Precursor Signals}

There seems to be a growing consensus that at least some electrical earthquake precursor signals are plausible (Bernard, 1992; Johnston, 1997), though the standard model does not provide a physically coherent concept. This is particularly relevant to those electrical signals that require charges to propagate through the rock volume (Dobrovolsky et al., 1989; Park, 1997a). Such signals are controversial (Geller, 1997; Michael, 1996; Pham et al., 1998). In some cases such as at Parkfield, California, along and across the San Andreas fault system no clear changes in the conductivity or any other electric effects related to seismicity have been identified (Park, 1997b).

Broadly speaking, electric and EM signals can be divided into three categories: (1) ground potentials, (2) EM emission and luminous phenomena, and (3) perturbations in the ionosphere.

1. Ground potentials can be generated as "streaming potentials" when saline water, moving through porous rocks, entrains ionic charges (Bernabé, 1998; Draganov et al., 1991; Morrison et al., 1989). Another way is through stress applied to rocks containing piezoelectric minerals such as quartz (Bishop, 1981; Finkelstein et al., 1973; Morat and Le Mouel, 1987, 1992), maybe also in the absence of piezoelectric minerals (Varotsos et al., 1997). The transmission of substantial stress over large distances has been debated (Geller, 1996; Huang and Ikeya, 1998; Masood, 1995).

(2) Low-frequency EM emissions reported from field observations have received some level of recognition as stated in reviews by Johnston and Parrot (1989), Park et al. (1993), and Johnston (1997). Such signals have been documented in connection with the 1989 Loma Prieta earthquake in California (Fraser-Smith et al., 1990), though their origin remains uncertain (Bernard, 1992, Merzer and Klemperer, 1997). EM emissions were also recorded during the 1989 Ito seismic swarm in Japan (Fujinawa and Takahashi, 1990). Detection of EM signals is pursued by groups in Japan, China, and 
countries of the former Soviet Union (Molchanov et al., 1992; Serebryakova et al., 1992) and in China (Zhijia, 1989). Puzzling and in part intertwined with folklore (Tributsch, 1983) are luminous phenomena reported to accompany some large earthquakes (Derr, 1973; Hedervari and Noszticzius, 1985), occasionally also weak events such as in 1988 in the Saint John region of Canada (Quellet, 1990). Earthquake lights were photographed during the 1966 earthquake at Matsushiro, Japan (Derr, 1986). Piezoelectricity from quartz-bearing rocks (Finkelstein et al., 1973) and sonoluminescence (Johnston, 1991) have been suggested though full laboratory confirmation seems to be still lacking.

(3) Ionospheric perturbations over earthquake regions have been observed during the 1964 Alaskan earthquake (Davis and Baker, 1965) and over parts of continental Asia (Bilichenko et al., 1990; Chmyrev et al., 1986; Drobgev et al., 1978; Galperin et al., 1985; Kustov and Liperovsky, 1988; Larkina et al., 1988), over Japan (Molchanov and Hayakawa, 1998a,b) such as over Kobe a few days before and after the January 1995 earthquake (Molchanov et al., 1998), and over Taiwan (Liu et al., 2000), including a few days before and after the September 1999 Chichi earthquake (Lee et al., 2000)

\subsection{Known Sources of Electric Charges in Rocks}

Known processes for the generation of electric charges are streaming potentials, piezoelectricity, triboelectricity/triboluminescence, spray and contact electrification.

Streaming potentials arise when weakly conducting liquids such as fuel or transformer oil are pumped through pipes and conduits (Oommen, 1988). In nature they occur when saline water moves through porous rocks, entraining solvated ions of one sign, while the charge-balancing counterions remain adsorbed to the rocks. The magnitude of the potentials is limited by the return currents flowing along the double layer (Bernabé, 1998; Draganov et al., 1991; Morrison et al., 1989).

Piezoelectricity describes the phenomenon that, when a stress is applied to certain crystals in certain crystallographic directions, opposite sides of the crystals become instantly charged (Finkelstein et al., 1973). Quartz is the only mineral in a piezoelectric symmetry class that is abundant in nature. In a stressed volume with quartz crystals in random orientation the piezopotentials tend to cancel.

Triboelectricity and triboluminescence describe phenomena that occur when crystals are abraded, indented or fractured. On an atomic level, rapidly moving dislocations create exciton pairs, i.e., electrons and holes, which luminesce upon recombination. Advancing fracture wedges cause charge separation on either the opposing sides (Enomoto and Hashimoto, 1990; Hadjicontis and Mavromatou, 1994; Yoshida et al., 1997, 1998; Brady and Rowell, 1986).

Spray electrification is a term used to describe the charging of water droplets by splashing or spraying (Loeb, 1958). Assuming that pore water evaporates suddenly when seismic waves disrupt surface rocks and cause intense local heating, spray electrification has been invoked by Lockner et al. (1983) as a possible mechanism to explain earthquake lights.

Contact electrification is a fundamental process that occurs whenever two dissimilar materials are brought in contact. It arises even in insulators because, however low their conductivity, they will always have a nonzero density of electronic charges. These charges define a Fermi level similar to the Fermi level in semiconductors. Upon contact, charges flow across the contact point until the Fermi levels are equalized. An exemplary experiment describing charge transfer between mica and quartz has been reported by Horn and Smith (1992).

However, none of the mechanisms or processes listed stands out as a candidate for bringing the electrical, electromagnetic and luminous phenomena together and present a coherent physical model. There seems to be only one way out: we have to consider the possibility that, despite decades of intense studies in laboratories worldwide, rocks may contain charge carriers that have not yet been properly identified. 


\subsection{Less Well-Known Source of Electric Charges in Rocks}

Electronic charge carriers in rocks are generally thought to be of minor concern because most minerals are good insulators. Electronic charge carriers can be electrons or defect electrons, the latter known as "holes." Both, electrons and holes can be associated with transition metal cations that change their oxidation states. In some minerals where an electronic exchange between neighboring transition metal cations can take place, hole conductivity occurs. However, except in cases where $\mathrm{Fe}^{2+}$ and $\mathrm{Fe}^{3+}$ are direct neighbors on crystallographically well-defined sites such as in magnetite, hole conductivity is rare. This is supported by the fact that even Fe-rich pyroxene and olivine show negligible electronic conductivity via their cation sublattices (Schock and Duba, 1985; Shankland, 1981).

What is hardly ever recognized is the fact that oxygen can also exist in two oxidation states: as $\mathrm{O}^{2-}$ in the common 2-oxidation state and as $\mathrm{O}^{-}$in the somewhat uncommon 1-oxidation state. Because textbooks invariably formulate mineral structures with the tacit assumption that oxygen is never anything but $\mathrm{O}^{2-}$, it is widely assumed that oxygen is locked into its 2 - oxidation state. However, given special local lattice conditions to be outlined below, $\mathrm{O}^{2-}$ can convert to $\mathrm{O}^{-}$. An $\mathrm{O}^{-}$in an $\mathrm{O}^{2-}$ matrix represents an electronic charge carrier, a hole, but it is different from the holes that reside on the cation sublattice. To underscore this difference an $\mathrm{O}^{-}$in the $\mathrm{O}^{2-}$ sublattice is designated as a "positive hole".

The remainder of this paper will be dedicated to positive holes and their possible role in generating electric signals in rocks.

\subsection{Nature of the Positive Holes}

Before addressing the question how oxygen in the -1 oxidation state is introduced into minerals and rocks, I would like to summarize what we believe to know about positive holes and about their propagation through rocks.

From a chemist's perspective, an $\mathrm{O}^{-}$is an oxygen anion with an incomplete valence shell, 7 electrons instead of the usual 8. An $\mathrm{O}^{-}$is a radical, and writing it as $\mathrm{O} \cdot$ is equally valid. If the $\mathrm{O}^{-}$is part of an $\mathrm{XO}_{4}{ }^{4+}$ complex $\left(\mathrm{X}=\mathrm{Si}^{4+}, \mathrm{Al}^{3+}\right.$, etc. $)$, it might be written either as $\mathrm{XO}_{4}{ }^{3+}$ or as $\mathrm{O}_{3} \mathrm{X}^{\circ}$. Being radicals, $\mathrm{O}^{-}+\mathrm{O} \cdot$ and $\mathrm{XO}_{4}^{3+}+\mathrm{gX}^{\circ \cdot}$ are unstable. They can react with each other by pairing up to form a positive hole pair, $\mathrm{PHP}$, chemically equivalent to a peroxy anion, $\mathrm{O}^{-}+\mathrm{O}^{-}=\mathrm{O}_{2}{ }^{2-}$ or a peroxy link $\mathrm{O}_{3} \mathrm{X}^{\mathrm{O}}{ }^{\circ}+{ }_{.} / \mathrm{XO}_{3}=\mathrm{O}_{3} \mathrm{X}^{\mathrm{OO}} \mathrm{XO}_{3}$. Such peroxy links can be thermodynamically stable, if they are associated with certain defect sites in the host mineral structure (King and Freund, 1984). Noteworthy is also the fact that the $\mathrm{O}^{-}-\mathrm{O}^{-}$distance is very short, generally less than $1.5 \AA$, about half the $\mathrm{O}^{2-} \ldots \mathrm{O}^{2-}$ distances in most mineral structures, 2.8-3.0 $\AA$. The shortness of the $\mathrm{O}^{-}-\mathrm{O}^{-}$bond implies a small partial molar volume of the peroxy-bound $\mathrm{O}^{-}$, and thus a tendency to be favored by high pressure.

From a physicist's perspective, the $\mathrm{O} \cdot$ or $\mathrm{O}_{3} \mathrm{X}^{\mathrm{O}}{ }^{\bullet}$ represents a positive hole as defined above, i.e. an electronic charge. A peroxy link then represents a self-trapped positive hole pair, PHP, immobile and electrically inactive (King and Freund, 1984). Most positive holes in minerals will be found in form of PHPs. Because the PHPs are electrically inactive and do not manifest themselves in any other easily recognizable way, the very presence of PHPs in minerals has been overlooked. However, PHPs may dissociate by breaking the $\mathrm{O}^{-}-\mathrm{O}^{-}$bond, thus releasing a positive hole charge carrier.

In the band model an insulator is defined as a material with a wide energy gap in which all valence band states are filled. No electrons can flow, and the conductivity is zero. Even in the presence of PHPs, the material remains an insulator. However, if a PHP dissociates, it introduces holes into the valence band, causing the insulator to become a p-type semiconductor. 
The propagation of positive holes through an oxide or silicate matrix can be discussed in terms of electron hopping. If one $\mathrm{O}^{2-}$ in a sea of $\mathrm{O}^{2-}$ changes to $\mathrm{O}^{-}$, it creates a situation whereby electrons from neighboring $\mathrm{O}^{2-}$ can hop onto the $\mathrm{O}^{-}$site. In first approximation the hopping frequency is controlled by phonons, e.g. by collisions with neighboring $\mathrm{O}^{2-}$, which typically occur at a frequency of the order of $10^{12} \mathrm{~s}^{-1}$. By multiplying the phonon frequency with the hopping distance, $\sim 3 \AA$, and considering a probability factor of $1 / 3$ that the electron hops in a given direction, we can estimate the maximum speed at which a positive hole could propagate, $100-300 \mathrm{~m} / \mathrm{s}$. Because the positive holes travel via the $\mathrm{O} 2 \mathrm{p}$-dominated valence band, they can cross grain boundaries without being scattered or annihilated.

\subsection{How are Positive Holes Introduced into Minerals and Rocks?}

In this section I discuss one way by which PHPs are introduced into mineral structures. As the basic reaction involves only the $\mathrm{O}^{2-}$ sublattice, the essential features can be described by looking at a simple oxide structure, $\mathrm{MgO}$.

When $\mathrm{MgO}$ crystallizes in the presence of $\mathrm{H}_{2} \mathrm{O}$, it incorporates small amounts of $\mathrm{H}_{2} \mathrm{O}$ in form of $\mathrm{OH}^{-}$, preferentially as $\mathrm{OH}^{-}$close to $\mathrm{Mg}^{2+}$ vacancy sites:

$$
\left(\mathrm{H}_{2} \mathrm{O}\right)_{\text {dissolved }}+\left(\mathrm{O}^{2-}\right)_{\text {structure }}\left(\mathrm{OH}^{-}\right)_{\text {structure }}+\left(\mathrm{OH}^{-}\right)_{\text {structure }} \text {. }
$$

It has been noted early (Martens et al., 1976) that such $\mathrm{OH}^{-}$pairs can undergo a previously unknown redox conversion whereby they split off molecular $\mathrm{H}_{2}$ and form two $\mathrm{O}^{-}$which in turn undergo self-trapping to a peroxy anion, $\mathrm{O}_{2}^{2-}$, e.g., a PHP:

$$
\left(\mathrm{OH}^{-}\right)_{\text {structure }}+\left(\mathrm{OH}^{-}\right)_{\text {structure }}=\left(\mathrm{H}_{2}\right)_{\text {structure }}+\left(\mathrm{O}_{2}{ }^{2-}\right)_{\text {structure }} \text {. }
$$

The presence of $\mathrm{H}_{2}$ molecules in $\mathrm{MgO}$ single crystals was confirmed by infrared spectroscopy (Freund and Wengeler, 1982). Evidence for PHPs was obtained by electrical conductivity (Freund et al., 1993), magnetic susceptibility, dielectric polarization measurements as well as by measuring other less direct physical properties (Freund et al., 1994). Though all these studies were done at ambient pressure, the fact that the partial molar volume of $\mathrm{O}^{-}$in the peroxy bond is much smaller than that of $\mathrm{O}^{2-}$ and $\mathrm{OH}^{-}$, suggests that pressure should favor the redox conversion of $\mathrm{OH}^{-}$pairs to $\mathrm{H}_{2}$ plus peroxy.

Silicate minerals, even those that are nominally anhydrous, also incorporate traces of $\mathrm{H}_{2} \mathrm{O}$ whenever they crystallize in $\mathrm{H}_{2} \mathrm{O}$-laden magmatic or metamorphic environments (Rossman, 1996). Initially they form $\mathrm{O}_{3} \mathrm{X}-\mathrm{OH}$ pairs:

$$
\left(\mathrm{H}_{2} \mathrm{O}\right)_{\text {dissolved }}+\left(\mathrm{O}_{3} \mathrm{X} / \mathrm{O} / \mathrm{XO}_{3}\right)_{\text {structure }}\left(\mathrm{O}_{3} \mathrm{X} / \mathrm{OH}_{\mathrm{HO}} / \mathrm{XO}_{3}\right)_{\text {structure }} \text { (3) }
$$

However, as the $\mathrm{OH}^{-}$pairs in $\mathrm{MgO}, \mathrm{O}_{3} \mathrm{X}-\mathrm{OH}$ pairs in silicates undergo a redox reaction whereby they also reduce two $\mathrm{H}^{+}$to $\mathrm{H}_{2}$ and oxidize two $\mathrm{O}^{2-}$ to $\mathrm{O}^{-}$, which in turn combine to form a peroxy link, typically between two adjacent $\mathrm{SiO}_{4}$ tetrahedra:

$$
\left(\mathrm{O}_{3} \mathrm{X} / \mathrm{OH}_{\mathrm{HO}} / \mathrm{XO}_{3}\right)_{\text {structure }}=\left(\mathrm{H}_{2}\right)_{\text {structure }}+\left(\mathrm{O}_{3} \mathrm{X} / \mathrm{OO} / \mathrm{XO}_{3}\right)_{\text {structure }} \text {. }
$$

Peroxy links in $\mathrm{SiO}_{2}$ have been studied theoretically by Edwards and Fowler (1982) and experimentally (Friebele et al., 1979; Freund and Masuda, 1991). The $\mathrm{O}^{-} \mathrm{O}^{-}$bond can break easily, releasing a positive hole. Continuing from (2) and (4) we have

$$
\begin{aligned}
& \left(\mathrm{O}_{2}^{2-}\right)_{\text {structure }}=\left(\mathrm{O}^{-}+\mathrm{O}^{-}\right)_{\text {structure }} \\
& \left(\mathrm{O}_{3} \mathrm{X} / \mathrm{OO} / \mathrm{XO}_{3}\right)_{\text {structure }}=\left(\mathrm{O}_{3} \mathrm{X} / \mathrm{O}^{\cdot} \cdot \mathrm{O}^{\circ} / \mathrm{XO}_{3}\right)_{\text {structure }}
\end{aligned}
$$


The dissociation of the peroxy bond according to (5) and (6) turns the insulator into a semiconductor. Since the positive holes are the only mobile charge carriers and no electrons are generated at the same time, the semiconductor is purely p-type.

In MgO, where PHPs are associated with $\mathrm{Mg}^{2+}$ vacancies (Freund et al., 1993, 1994), the two positive holes compensate for the charge of the missing $\mathrm{Mg}^{2+}$ cation. When the PHP dissociates and one positive hole becomes a mobile charge carrier, the other positive hole stays behind at the $\mathrm{Mg}^{2+}$ vacancy site but is immobile because $\mathrm{Mg}^{2+}$ vacancies only diffuse at temperatures above $700^{\circ} \mathrm{C}$. Similar analogous arguments can be made for $\mathrm{PHP}$ in $\mathrm{SiO}_{2}$ or feldspars, where the $\mathrm{XO}_{4}{ }^{4-}$ are linked at all four corners by $\mathrm{O}_{3} \mathrm{X}-\mathrm{O}-\mathrm{XO}_{3}$ bonds. After $\mathrm{O}_{3} \mathrm{X}^{\prime} \mathrm{OO} \mathrm{XO}_{3}$ dissociation according to equation (6) one $\mathrm{XO}_{4}{ }^{4+}$ and one $\mathrm{XO}_{4}{ }^{3+}$ remain. This configuration represents a negative charge that is stationary compared to the positive hole which is mobile.

For understanding the experiments to be described below it is important to note that, when positive holes are the only mobile charge carriers, their mutual electrostatic repulsion forces them to the surface. This outward flow of positive charges continues until balanced by the electric field that builds up between the surface and the negatively charged bulk (King and Freund, 1984). For the impact experiments this has two consequences: (1) The magnitude of the positive surface potential becomes a function of the dielectric contrast between the bulk and air, $\sim 1$. Assuming that the rock has a dielectric constant $\sim 10$ the calculated flat surface potential in vacuum, $=1$, is $+420 \mathrm{mV}$. ( $R$ The electric field at the surface becomes a function of the concentration of positive holes in the bulk. When their concentration increases from $10 \mathrm{ppm}$ to $100 \mathrm{ppm}\left(10^{17}\right.$ to $\left.10^{18} \mathrm{~cm}^{-3}\right)$, the surface field increases from $1.2 \times 10^{5}$ to $4 \times 10^{5} \mathrm{~V} / \mathrm{cm}$, high enough to consider dielectric breakdown of the surrounding air.

\subsection{Why Impact Experiments?}

The impact experiment described here were not undertaken in order to simulate as closely as possible a specific natural condition, but to achieve two basic goals. First, to demonstrate that positive hole-type charge carriers can be generated from pre-existing, dormant PHP precursors. Second, to gain insight into the somewhat surprising properties of these charge carriers as they propagate through the rocks. The idea came from prior single crystal fracture experiments, using $\mathrm{MgO}$ as a well-characterized oxide material (Dickinson et al., 1986) and olivine. These experiments suggested that the acoustic waves or dislocation movements generated by the brittle fracture were enough to cause PHP dissociation according to equations (5) and (6).

If the weak acoustic waves generated by brittle fracture are capable of activating the positive holes, the question arose whether microfracturing in rocks prior to an earthquake might also activate positive holes, at least in a small volume around the microfracture event. Likewise, if the stresses cause plastic deformation (creep) of the rocks and, hence, dislocation movements, positive holes are likely to be activated. Assuming that microfracturing and creep become pervasive as the tectonic stresses reach critical levels, activation of positive holes and their outflow from the stressed volume could represent the long-sought charge generation mechanism responsible for preseismic electric and electromagnetic signals that have been reported from the field prior to earthquake activity.

\section{Experimental Procedures}

\subsection{Low-Velocity Impact Experiments}

Low-velocity impact experiments were conducted as described earlier (Freund and Borucki, 1999), using stainless steel, $1 / 8$-inch to $1 / 4$-inch $(3.1-6.3 \mathrm{~mm})$ diameter projectiles at close to $100 \mathrm{~m} / \mathrm{s}$ delivering $0.06-0.43 \mathrm{~J}$ kinetic energy to cylindrical rock cores, 3/4-inch $(20 \mathrm{~mm})$ diameter and up to 4 - 
inch $(100 \mathrm{~mm})$ long. The shots left small whitish marks on the front face of the rock cores and led to chipping and fracture.

The sensors included: (1) axially mounted magnetic pick-up coils $(2 \mathrm{~cm}$ wide, 3000 turns, 30 gauge magnetic wire), (2) photodiodes placed either so as to "see" the front face, or at the end inside tubes to "see" the rim or the flat face, (3) a ring capacitor near the front, (4) a plate capacitor at the back, and (5) ring electrodes attached directly onto the rock surface, but no strain gauges. Currents flowing from the electrodes into the rocks were measured by the voltage drop across a 2.4-M_resistance. The grounding point was about $2 / 3$ down the length of the cores. $200 \mathrm{MHz}$ digital 4-Channel Tektronix oscilloscope was used for data acquisition.

\subsection{Medium Velocity Impact Experiments}

Medium velocity impact experiments were carried out at the NASA Ames Vertical Gun Range (AVGR) in a $3 \mathrm{~m}$ wide, $2.5 \mathrm{~m}$ high vacuum chamber, evacuated to $\sim 4 \mathrm{mbar}$ as described earlier (Freund, 2000). The vacuum enclosure shielded the sample from the Earth's magnetic field. The samples were $25 \times 25 \times 20 \mathrm{~cm}$ blocks of the Barre granite. The projectiles were $1 / 4$-inch $(6.3 \mathrm{~mm}$ ) diameter Al spheres at vertical incidence. To remove adventitious electrostatic charges which the projectiles might accumulate during acceleration in or exiting from the gun barrel, the $\mathrm{Al}$ sphere passed through a thin, grounded Al foil.

The rock was instrumented with three types of sensors:

1. Three magnetic pick-up coils on $26 \times 26 \mathrm{~cm}$ square wooden frames with 300 windings of 30 gauge magnet wire, one positioned $10 \mathrm{~cm}$ above the plane of impact to record primarily the emission from the plasma plume (Crawford and Schultz, 1991), the two other-coils positioned slightly below midheight and near the bottom of the block, respectively. Though the spacing between the coils was less than their diameters, self-induction was found to be negligible.

2. Three capacitors, consisting of $22 \mathrm{~cm}$ long, $20 \mathrm{~mm}$ wide pieces of $0.05-\mathrm{mm}$-thick electrical insulation tape onto which were glued 20 -cm-long, 12-mm-wide strips of $\mathrm{Cu}$ tape. The location of the capacitor sensors will be shown in the figures below.

3. Two electrodes made of adhesive conductive $\mathrm{Cu}$ tape, $20 \mathrm{~cm}$ long, $12 \mathrm{~mm}$ wide, glued directly onto the rock to provide direct contacts. The electrodes were connected to ground through $2.4-\mathrm{M}_{\text {- }}$ resistances across which the voltage drop was measured. The location of the electrodes will be shown in the figures below.

The rock rested on a $1.5-\mathrm{cm}$-thick Al metal plate, connected to the oscilloscope ground.

For data acquisition two four-channel digital oscilloscopes were used in parallel: (1) a $200-\mathrm{MHz}$ Tektronix oscilloscope, model $420 \mathrm{~A}$, to record the signals from the three pick-up coils and one of the two electrodes; (2) a 500-MHz Tektronix oscilloscope, model 756, to record the signals from the three capacitors and the other electrode. Data acquisition was triggered through the AVGR electronics. Both oscilloscopes stored the data from the trigger point minus $5 \mathrm{~ms}$ until the buffer was full, typically $\sim 30$ ms after impact.

All shots were fired at normal incidence. Shots 1 and 3 served to test all channels and tune the settings so that the signals recorded by each sensor were strong enough and clearly resolved but not offscale. Shot 2 was lost due to a triggering error. Shot 4 at $1.46 \mathrm{~km} / \mathrm{s}$ produced usable data on all eight channels, while shot 5 at $1.79 \mathrm{~km} / \mathrm{s}$ gave usable data on five of the eight channels. The shots, all fired at the same spot, led to the excavation of a near-cylindrical hole, $\sim 10 \mathrm{~mm}$ wide, growing to a depth of $\sim 50 \mathrm{~mm}$ and possibly deeper after shot 5 . Shot 6 at $4.45 \mathrm{~km} / \mathrm{s}$ gave usable data on all eight channels but caused three fissures to appear at roughly $120^{\circ}$ on the surface of the block. Shot 7 at $5.64 \mathrm{~km} / \mathrm{s}$ caused catastrophic fracture into three segments. 


\subsection{Samples}

The low-velocity experiments were conducted with two rocks, air-dry: (1) a whitish-gray speckled gabbro with phaneritic texture containing $\sim 80 \%$ whitish plagioclase (bytownite with $88 \%$ An) and $\sim 20 \%$ large, dark, elongate pyroxene (clinopyroxene « orthopyroxene) with thin augite exsolution lamellae, and (2) a dark, almost black diorite with phaneritic texture containing abundant orthopyroxene, often with very fine exsolution lamellae, clouded plagioclase (labradorite with $67 \% \mathrm{An}$ ), magnetite, minor hornblende and biotite and very minor $\mathrm{K}$-feldspar and quartz. When impacted several times, the gabbro cores chipped more easily than the diorite cores.

The medium-velocity experiments were conducted with air-dry granite from Barre, Vermont, roughcut with a diamond saw but not polished. The granite is coarse-grained, light gray with approximately $35 \%$ quartz, $55 \%$ sodium and potassium feldspar, minor plagioclase, plus some biotite and hornblende. The rock shows no sign of texture that could lead to a preferred orientation of its quartz crystals.

\section{Results}

\subsection{Low-Velocity Impacts}

When a steel ball hits the rock, several effects occur in rapid succession, variable from shot to shot, but displaying a recurring pattern. Figure la shows an experiment with a gabbro core, equipped with a photodiode at the front face (channel 4), a ring capacitor near the front (channel 2), and a plate capacitor at the back end (channel 3 ).

The moment of impact is marked by a short $(10 \mu \mathrm{s})$, weak light blip (vertical arrow). About $150 \mu \mathrm{s}$ after impact the ring capacitor, $10 \mathrm{~mm}$ from the impact point, begins to record a positive voltage that rises to above $+400 \mathrm{mV}$. About $250 \mu \mathrm{s}$ after impact the back end plate capacitor, $70 \mathrm{~mm}$ from the impact point, begins to show a positive voltage that rises to $+40 \mathrm{mV}$. When the front ring capacitor voltages exceeds +400 to $+450 \mathrm{mV}(\sim 450 \mu$ s after impact), light is emitted, much stronger and longer lasting than that at impact. This effect will be called the "delayed light." At the same moment the front capacitor voltage breaks down and may even go negative.

The same effects are even more clearly displayed in Figure 1b where a diorite core was used. Only the front capacitor voltage (channel 3) and the light emission (channel 4) are shown. The impact light blip was not picked up, probably because the ricocheting steel ball blocked the light path. The dotted line on channel 3 traces the voltage at the front capacitor that may have reached values well above +400 $\mathrm{mV}$ without the intervening delayed light emission, causing it to break down abruptly. As in Figure la, when the delayed light emission occurs, the front capacitor voltage collapses and turns briefly negative. It recovers to around $+300 \mathrm{mV}$ and decays in an irregular manner that may contain some low frequency components.

In Figure 1c, also with the diorite core, the front ring capacitor was replaced by a magnetic pick-up coil, while leaving the photodiode at the front and the plate capacitor at the end in place. At impact, the coil registers an EM emission. Its intensity increases slightly at the delayed light emission. This is a recurring feature that is often observed and some times quite pronounced.

In Figure 2 the magnetic pick-up coil (channel 2) was moved along the axis and a contact ring electrode (channel 1) was installed at the front end, leaving the back end plate capacitor in place. Channel 3 records the back end capacitor voltage. Channel 4 records the light emission. What is different with respect to the configurations in Figures $2 a-2 c$ is that the rock core now is grounded at two places, first via the electrode across the 2.4-M_resistor and second at the grounding point about $2 / 3$ down the length of the core. At impact, a $10-20 \mu$ s light blip occurs plus a sharp onset of EM emission that continues for several ms. The front end electrode records a positive voltage $\sim 200 \mu$ s after impact, indicating that the electrode begins to inject electrons into the rock. The electron injection increases steeply with the voltage signal rising beyond $+500 \mathrm{mV}$ and eventually going off scale. The 
delayed light emission sets in sharply $400-420 \mu$ s after impact. At the same moment the positive voltage recorded by the back end capacitor (which had just started to rise) begins damped oscillations in the range of $8 \mathrm{kHz}$.

Such $\mathrm{kHz}$ oscillations at the back end capacitor are seen in all experiments where the rock was grounded at more than one place. Examples are given in Figures $4 \mathrm{a}$ and $4 \mathrm{~b}$ with a diorite core equipped with three ring electrodes in direct contact with the rock surface (channels 1-3), the back end capacitor, and the front photodiode. Because only four recording channels were available, we conducted two shots under otherwise identical conditions, switching channel 4 from the back capacitor (Figure 3a) to the photodiode (Figure $3 b$ ).

In Figure 3a the moment of impact is moved off-scale to the left. The three electrode voltages rise simultaneously, indicating the time when all three electrodes begin to inject electrons into the rock. As the voltage at the first two electrodes reaches maximum and begins to break down (presumably because of the on-set of a delayed light emission), the voltage of the back end capacitor begins to oscillate at about $40-45 \mathrm{kHz}$, which is higher than in the case depicted in Figure 2. The oscillations decay over $<1$ ms. Concurrently, a $40-45 \mathrm{kHz}$ ripple appears on the voltage signal recorded from the third electrode, closest to the grounding point, suggesting some kind of coupling between the electron injection and the electric field at the rock surface sensed by the back end capacitor.

The second shot is depicted in Figure $3 \mathrm{~b}$ where channel 4 was used for the photodiode. A 20-30 $\mu \mathrm{s}$ impact light blip is clearly identified. It is followed $250 \mu$ s later by the simultaneous build-up of the voltage at the three contact electrodes, signaling electron injection into the rock. About $400 \mu \mathrm{s}$ later a sudden, intense delayed light emission occurs. At this exact moment, the voltage at the first and second electrode voltage begins to break down. The third electrode, closest to the grounding point, registers the same faint $40-45 \mathrm{kHz}$ ripple as in Figure 3a. This indicates that when the rock is grounded and a positive charge appears on its surface, electron injection occurs, often in an oscillatory fashion. This effect will be more clearly demonstrated in the course of the medium velocity experiments. The ensemble of the results obtained so far suggest that the low-velocity impacts, even those with the lightest steel balls, generate electrical charge carriers in the rocks. Their sign is positive. There seems to be no threshold for the appearance of these charge carriers, though the range of the kinetic energy deposited into the rocks in this part of the impact experiments, 0.06-0.4 Joules, is small and may have precluded identification of such a threshold. Furthermore, the observations indicate that the charge carriers spread through the cores at a relatively high speed, in the range of $100-300 \mathrm{~m} / \mathrm{s}$. Such high speed can only be associated with electronic charges, and since they carry a positive sign, they are identified as defect electrons.

When the integrated visible light intensity as registered by the front end photodiode is plotted versus the kinetic energy of impact, a linear relationship seems to be obtained as demonstrated in Figure 4. This is consistent with the idea that the volume in which PHPs are activated and, hence, the number of positive hole charge carriers generated increase linearly with impact energy.

Another experiment was set up to test whether the rock becomes conductive as the cloud of charge carriers propagates. The sample consisted of a rectangular piece of diorite, $15 \times 15 \times 40 \mathrm{~mm}$, with polished ends onto which 5-mm-diameter gold electrodes were sputtered; $25 \mathrm{~V}$ were applied across the long dimension, $40 \mathrm{~mm}$, while the block was impacted from the side, normal to the applied electric field, using a $1 / 8$-inch steel ball. The current flowing through the rock was determined by measuring the voltage drop across a $2.4-M_{\text {_ }}$ resistance $R$ (Channel 3). In addition, the light emission the front face and the EM emission were recorded (channel 1 and channel 4, respectively).

A sharp onset of EM emission marks the impact as seen in Figure 5. About $150 \mu \mathrm{s}$ later, the voltage across the resistance $\mathrm{R}$ rises, indicating a current flowing through the rock, reaching $300 \mathrm{mV}$ within 150 $\mu \mathrm{s}$, and ending abruptly with a sharp light pulse accompanied by $\mathrm{a}<10 \mu \mathrm{s}$ long EM pulse. Within 200 $\mu$ s the sign of the voltage reverses, indicating a reversal of the current. Thereafter, both voltage signals 
and EM signals reverberate for a few milliseconds. These observations suggest that the charge carriers generated locally by the impact propagated outward from their source volume, causing the rock to become momentarily conductive and to support a cross current between the Au electrodes.

\subsection{Medium-Velocity Impacts}

With the medium velocity impacts three questions were addressed: (1) Will the granite also generate positive hole charge carriers? (2) Is the mode of activation different at high kinetic energies? (3) How does the presence of piezoelectric quartz affect the measurements?

Figure 6 shows data recorded on the 200-MHz oscilloscope during shot $4(1.46 \mathrm{~km} / \mathrm{s})$. The locations of the coils and the top contact electrode are indicated in the inset. The approximate time of impact is marked by the dotted line. Channels 1-3 registered signals from the top, middle and bottom pick-up coils at 200,100 , and $50 \mathrm{mV} / \mathrm{div}$, respectively. Channel 4 registered the signal from the top contact electrode at $200 \mathrm{mV} /$ division.

All three coils "see" an EM emission. The signal recorded by the plasma coil is relatively weak and lasts for less than $500 \mu \mathrm{s}$, suggesting that the impact did not produce the type of intense plasma plume known from high velocity or hypervelocity impacts (Crawford and Schultz, 1991). The EM signal recorded by the middle coil lasts about $500 \mu$ s and that recorded by the bottom coil for about $1.5 \mathrm{~ms}$.

Figure 7 a shows the same EM signals as in Figure 6 at higher time resolution. Taking the first EM spike recorded by the plasma coil as the moment of impact, the middle coil and bottom coil begin registering EM emissions at the times as marked by the arrows, about $35 \mu$ s and $65 \mu$ s after impact. The delayed onset indicates that the middle and bottom coils register an electric charge moving through the block much faster than after the low velocity impact experiments, i.e. at a speed commensurate with the velocities of the $P$ or $S$ waves, about 6 and $3.4 \mathrm{~km} / \mathrm{sec}$ respectively.

The same $\sim 35$ and $65 \mu$ s delay of the signals from the middle and bottom coils was observed during shot 6 at $4.45 \mathrm{~km} / \mathrm{s}$ as shown in Figure $7 \mathrm{~b}$, indicating that the time delay is not a function of the impact velocity. The envelope of the plasma EM emission signal is quite regular, possibly because the previous shots had by then excavated a hole $\sim 10 \mathrm{~mm}$ wide and $70 \mathrm{~mm}$ deep into the granite block which focussed the plasma plume into a narrow fountain. Shot 6 resulted in three vertical fissures, running through the granite block at $\sim 120^{\circ}$ with respect to each other. These fissures began to form after the 2 $\mathrm{ms}$ time window presented in Figure $7 \mathrm{~b}$. However, they did not yet lead to a disintegration of the block.

An example of EM emission when the rock fractures after the impact is shown in Figure 7c, presenting data from shot 7 at $5.64 \mathrm{~km} / \mathrm{s}$. The block broke into three sections along the fissures preformed during shot 6 . The data from the bottom coil were lost. The EM emission recorded by the plasma coil and the middle coil is shown on two different timescales, over $30 \mathrm{~ms}$ in the main panel and over $2.5 \mathrm{~ms}$ in the inset. The plasma coil registered an intense EM emission immediately after impact with reverberations that lasted for about $2 \mathrm{~ms}$, maybe because the plasma jet emitted upon impact became entangled in the cracks that opened as the rock began to disintegrate. The middle coil registered its first signal with the expected time delay of $\sim 65 \mu \mathrm{s}$, followed by multiple bursts of EM emission, at 5,20 , and $26.5 \mathrm{~ms}$, probably all associated with the opening and propagation of the cracks.

While the EM emissions recorded by the pick-up coils indicate rapidly moving charges generated upon impact, the three capacitive sensors register the potential appearing on the granite surface. Figure 8 a shows the voltage response of the three capacitive sensors that were all on one side of the block as depicted in the inset plus the voltage registered by the bottom electrode, $<1 \mathrm{~cm}$ from the grounded metal base. The time of impact is marked by the dotted line.

The response of the capacitive sensors is significantly different from that seen after low velocity impacts. Now, the signals travel much faster, arriving within $65 \mu \mathrm{s}$ or less at the three sensor locations $\sim 15,18$, and $25 \mathrm{~cm}$ from the point of impact, respectively. However, instead of a smoothly rising 
positive voltage as before, the signals begin with a sequence of short pulses, lasting for 20-30 $\mu$ s. After $\sim 500 \mu \mathrm{s}$, e.g. at a time when the EM emissions have largely ended as shown in Figure 7a, a strong positive charge builds up on the entire rock surface. Concurrently, a new kind of oscillations takes over, their amplitudes increasing from the top to the bottom capacitive sensors.

In Figure $8 \mathrm{~b}$ the rapid pulses during the first $500 \mu \mathrm{sec}$ after impact are replotted. The vertical line marks the time of impact as derived from the onset of the EM emission as seen by the plasma coil (Figure 7a). The inset in the upper right shows the $P$ and $S$ wave velocity for Barre granite as a function of confining pressure (Christensen, 1982). In vacuum, the $P$ and $S$ wave velocities should all into the range of $5.5-6 \mathrm{~km} / \mathrm{s}$ and $3-3.4 \mathrm{~km} / \mathrm{s}$, respectively.

Because the granite contains quartz, the propagation of the $P$ and $S$ waves should be accompanied by transient piezoelectric voltage effects. Indeed, shortly after impact, all three capacitive sensors begin to register a series of short voltage pulses, 20-30 $\mu$ s wide. The distances from the point of impact to the three $20-\mathrm{cm}$-long, $1-\mathrm{cm}$-wide capacitor stripes vary from 12.5 to $17 \mathrm{~cm}$ (average $\approx 15 \mathrm{~cm}$ ) for the top sensor, from 15 to $23 \mathrm{~cm}$ (average $\approx 19$ ) for the middle sensor, and from 22 to $31 \mathrm{~cm}$ (average $27 \mathrm{~cm}$ ) for the bottom sensor. The arrival times of the first voltage pulse at the three sensor locations are delayed by $\sim 20-30,30-40$ and $50-65 \mu$ s, respectively.

These delays suggest a wave traveling down through the rock at a speed close to that of the $P$ wave, at about $6 \mathrm{~km} / \mathrm{s}$. The signals, in particular those from the top and middle sensors, show a rapid inversion from positive to negative voltages such as one would expect from the compression and rarefaction phases of a passing $P$ wave. Marking this first train of voltage pulses by solid circles, the solid line in Figure $8 \mathrm{~b}$ traces the propagation of the $\mathrm{P}$ wave. Beginning with the second voltage pulse registered by the top sensor, a second line (dashed) can be drawn through a second, slower train of voltage pulses that appears to correspond to the $S$ wave propagating at about $3 \mathrm{~km} / \mathrm{s}$. Finally, the open circles and dotted lines in Figure $8 \mathrm{~b}$ tentatively mark signals that may be caused by the reflected $\mathrm{P}$ and $\mathrm{S}$ waves.

If these trains of voltage pulses recorded by the three capacitive sensors are indeed due to a piezoelectric response from quartz at the rock surface, these signals should last only as long as the transient stresses exerted by the traveling $\mathrm{P}$ and $\mathrm{S}$ sound waves. Therefore, the piezoelectric signals should rapidly decay. Assuming that the sound waves reverberate in the granite block over two to three reflection cycles, each lasting $<100 \mu$ s for the $\mathrm{P}$ wave and $<200 \mu$ s for the $S$ wave, the piezoelectric signals are expected to decay within 500-700 $\mu$ s. This time window agrees rather well with the duration of the EM emission recorded by the middle coil as shown in Figures $8 \mathrm{a}$ and $8 \mathrm{~b}$, suggesting that a large component of the EM emission comes from transient piezoelectric voltage pulses that are created by the reverberating $P$ and $S$ waves.

However, Figure 8a also shows that, while the piezosignals fade away within $700-800 \mu$ s after impact, the top capacitive sensor continues to register a positive potential that builds up over the same time, indicating a positive surface charge. The potential reaches about $+400 \mathrm{mV}$ within $700-800 \mu \mathrm{s}$ and decays over the next $2 \mathrm{~ms}$. The middle and the bottom capacitive sensors register $+380 \mathrm{mV}$ and +300 $\mathrm{mV}$, respectively. These voltages are overprinted by amplitude variations that become more intense from top to bottom and are in phase.

Overall, with some additional features and complications, the medium-velocity impacts yield voltage signals that resemble those recorded after the low-velocity impacts. In both cases there is evidence that positive charge carriers are generated or activated by the impacts or rather by the acoustic waves resulting from the impact events. In the case of low-velocity impacts the charge generation seems to be limited to a small rock volume close to the impact point. From there the charges propagate as a cloud through the rock volume. In the case of the medium-velocity impacts the higher kinetic energy carried by the impactors produce sound waves of higher amplitudes. Apparently, after the reverberations of the sound waves had died down, the entire rock volume was left with activated positive holes which 
then spread outward toward the surface to reach a new equilibrium before deactivating, probably by some recombination or trapping mechanism.

The amplitudes of the oscillations that overprint the capacitive voltage signals increase the closer the sensor is to the grounded metal base plate. The oscillations are in-phase, suggesting that they are due to electric field variations occurring simultaneously at all three sensor locations. By contrast, the oscillations of the capacitive sensors are out-of-phase with those of the contact electrodes. The question arises: What is the cross relationship between these two sets of signals?

Figure 9 plots the voltage of the bottom capacitive sensor and voltage of the bottom contact electrode, the former representing the surface potential and the latter deriving from the current injected into the rock. As seen in the insets in Figure 8, both sensors are close to the grounded metal base plate, $\sim 1 \mathrm{~cm}$, but on opposite sides of the block. The oscillations are mostly out-of-phase, especially from 1 ms onward, suggesting that, when the potential at the rock surface exceeds the barrier height, the contact electrode will begin injecting electrons. This in turn causes the potential at the rock surface to break down, shutting off the electron injection. As long as positive holes still arrive at the surface from within the bulk, the potential recovers. However, as the process slows down, the frequency of the oscillations decreases. Eventually, when the surface potential can no longer reach or exceed the barrier height, electron injection shuts off entirely.

\section{Discussion}

Low- and medium-velocity impact experiments seem to be a valuable tool to study the activation of charge carriers in rocks. Through timing the impacts and measuring the evolving processes with microsecond to millisecond resolution, the response of rocks can be studied in ways that are not possible with rock samples placed under slowly increasing compressive or shear stress. The insights gained may shed light on a range of phenomena associated with earthquake activity - electrical, electromagnetic, and luminous - that have a long tradition in folklore from many parts of the world and have been reported in the scientific literature.

\subsection{Recapitulation}

When common igneous rocks are subjected to the sudden stress load of an impact, they generate highly mobile charge carriers. The charge carriers are electronic in nature. They spontaneously appear upon impact, propagate and disappear after a few milliseconds. They can be reactivated by repetitive impacts. Apparently, these charge carriers already exist in the rocks in their "as received" state, albeit in an electrically inactive, dormant form. At low kinetic impact energy they are activated in a small volume close to the impact point. At higher kinetic impact energies they seem to be activated in the wake of the sound waves, filling momentarily the entire rock volume.

Since the charge carriers are electronic in nature and positive, they can only consist of holes as argued in section 3 , specifically of positive holes, e.g. defect electrons in the $\mathrm{O} 2 \mathrm{p}$-dominated valence band of the constituent silicate minerals, which are equivalent to $\mathrm{O}^{-}$in an $\mathrm{O}^{2-}$ matrix. A likely dormant precursor form are positive hole pairs, PHPs, chemically equivalent to peroxy links, $\mathrm{O}^{-}-\mathrm{O}^{-}$or $\mathrm{O}_{3} \mathrm{X} / \mathrm{OO} / \mathrm{XO}_{3}$ with $\mathrm{X}=\mathrm{Si}^{4+}, \mathrm{Al}^{3+}$, etc. The PHPs are believed to be introduced into nominally anhydrous minerals through the dissolution of small amounts of $\mathrm{H}_{2} \mathrm{O}$ and subsequent redox conversion of $\mathrm{O}_{3} \mathrm{X}-\mathrm{OH}$ pairs into $\mathrm{O}_{3} \mathrm{X} / \mathrm{OO}_{\mathrm{XO}}$ plus $\mathrm{H}_{2}$ as described by equations (1)-(4).

\subsection{Why have they not been observed earlier?}

On the basis of the available impact data all three types of rocks - gabbro, diorite and granite - display a behavior that is consistent with the activation of positive hole charge carriers. At the same time these 
rocks are good insulators when dry and at ambient temperature. Their electrical conductivity has been studied under a wide variety of conditions: temperature, atmosphere, confining pressure etc. (e.g. Shankland and Ander, 1983). However, none of these studies, though carefully conducted, seem to have produced evidence for the highly mobile electrical charge carriers that show up so prominently in the impact experiments described here.

This is reminiscent of a situation encountered during a study of the model insulator $\mathrm{MgO}$ and its electrical conductivity as a function of temperature (Freund et al., 1993). $\mathrm{MgO}$ had long been considered an excellent insulator up to temperatures above $700^{\circ} \mathrm{C}$, the onset of ionic conductivity. There were hints of an unusual orders-of-magnitude increase in conductivity in the $400-700^{\circ} \mathrm{C}$ interval but those hints were not pursued because it could be demonstrated that the enhanced conductivity was confined to the surface. It looked suspiciously like some sort of contamination or "dirt." Indeed, upon heating $\mathrm{MgO}$ crystals long enough to temperatures well above $700^{\circ} \mathrm{C}$, the enhanced surface conductivity can be shown to disappear, suggesting that the contamination or "dirt" had been successfully removed. This settled the question for good in the minds of most investigators. A similar orders-of-magnitude enhancement of the electrical conductivity below $700^{\circ} \mathrm{C}$ was observed during the study of single crystals of upper mantle olivine by Constable and Duba (1990). As in the case of $\mathrm{MgO}$, the conductivity seemed confined to the crystal surface. Upon prolonged heating in a $1: 1$ $\mathrm{CO} / \mathrm{CO}_{2}$ gas mixtures, the enhanced conductivity disappeared, leading to the suggestion that a thin but conductive carbon film had vapor-deposited on the sample surface from the $\mathrm{CO} / \mathrm{CO}_{2}$ gas.

Meanwhile evidence had accumulated that $\mathrm{MgO}$ and probably also olivine crystals from the upper mantle contain peroxy anions and peroxy links respectively, e.g. PHPs, that are capable of releasing positive hole charge carriers (King and Freund, 1984; Freund et al., 1993; 1994). The behavior of these positive holes is unlike that of other charge carriers and unlike that expected for carbon films. The property that is most easily misunderstood is due to the mutual repulsion of positive holes in the bulk which effectively "pushes" them to the surface, causing an enhanced surface conductivity. However, positive holes are nothing but $\mathrm{O}^{-}$in an $\mathrm{O}^{2-}$ matrix and therefore highly oxidizing radicals. Their behavior, i.e. their chemical reactivity during a conductivity experiment chemical reactivity needs to be taken into account. Allowing positive hole charge carriers at a surface to react with $\mathrm{CO} / \mathrm{CO}_{2}$ gas mixtures at $700^{\circ} \mathrm{C}$ leads to their annihilation, $\mathrm{CO}+2 \mathrm{O}^{-}=\mathrm{CO}_{2}+\mathrm{O}^{2-}$. Using the reaction $\mathrm{CH}_{4}+\mathrm{O}^{-}=$ $\cdot \mathrm{CH}_{3}+\mathrm{OH}^{-}$, where $\cdot \mathrm{CH}_{3}$ represents the gas-phase methyl radical, the $\mathrm{O}^{-}$can in fact be titrated off the sample surface. Because of these unusual physical and chemical properties of the positive holes outlined above their potentially very important role as charge carriers in rocks has not been recognized, despite of many careful laboratory studies.

\subsection{Positive Holes and Electron Injection}

In the case of low velocity impacts, the activation seems to be confined to a small volume near the impact point. The light blip marking the time of impact is probably due to triboluminescence, coming from electron-hole pairs generated by rapidly moving dislocations and their radiative recombination. From the small source volume the positive holes propagate outward as a charge cloud, causing positive potentials, EM and delayed light emission.

In the case of medium velocity impact experiments, the activation of positive hole charge carriers most likely occurs in the wake of the $\mathrm{P}$ or $\mathrm{S}$ waves. After the initial piezoelectric signals from quartz crystals that were stressed by the propagating $P$ and $S$ waves have died down, the capacitive sensors register a pervasive positive charge that persists for $\sim 3 \mathrm{~ms}$, while the contact electrodes indicate pulses of electrons flowing into the rock. 
Figure 10 sketches the sequence of events following a medium velocity impact with special emphasis on the surface potential recorded by the capacitive sensors and the current measured by the contact electrodes. In (a) the impact occurs, sending acoustic waves into the rock. In (b) positive holes, symbolized by dots, become momentarily activated in the entire rock volume. In (c), due to mutual repulsion, the positive holes diffuse outward toward the surface as indicated by the arrows. In (d) the positive potential that builds up at the surface leads to the injection of electrons, e', from the grounded metal base plate and the contact electrodes.

Figure 11 follows the evolution of the signals in greater detail. Figure 11 (top) shows the granite block with the bottom capacitive sensor recording the voltage $V$ and the bottom electrode recording the current I near the grounded base plate. On the left the initial state is depicted, assuming that after passage of the acoustic waves, the rock volume is uniformly filled with mobile positive hole charge carriers. The initial state evolves toward the final state depicted on the right where the positive holes have come to the surface forming a positively charged surface layer balanced by a negatively charged interior. If the speed of propagation of the positive holes is of the order of $100 \mathrm{~m} / \mathrm{s}$, a block of granite measuring $25 \times 25 \times 20 \mathrm{~cm}$ can be expected to reach the final state within $\sim 1-2 \mathrm{~ms}$, as indeed observed.

The three panels in Figure 11 (middle) depict from top to bottom: (1) the surface potential as it would be expected to build up during the transition from initial to final state if the granite block were electrically isolated; the dashed line at $V_{i}$ marks the threshold voltage above which electron injection can occur across the metal-to-insulator contact; (2) the surface potential as recorded by the capacitive sensor, and (3) the injection current as recorded by the contact electrode. Each time the surface potential reaches the threshold value of $\mathrm{V}_{\mathrm{i}}$, electron injection occurs and the potential breaks down. This in turn shuts off the electron injection and allows the surface potential to recover. As the final state is approached, the time needed to reach $\mathrm{V}_{\mathrm{i}}$ becomes longer, causing the oscillations to slow down. In Figure 11 (bottom) the frequency of oscillation (in $\mathrm{kHz}$ ) for the surface potential (circles) and for the injection current (squares) is plotted versus time. The frequency decreases. After $\sim 3 \mathrm{~ms}$ the oscillations ceased altogether, suggesting that the positive holes reaching the rock surface no longer build up a sufficiently high potential for electron injection to occur.

\subsection{Application to Electrical Phenomena Related to Earthquakes}

As indicated at the beginning, the experiments reported here were not set up to simulate specific conditions encountered in connection with earthquake activity. Rather, they were designed to test whether igneous rocks such as gabbro, diorite and granite contain dormant charge carriers, which can be activated by microfracture and seismic waves.

On the basis of earlier work quoted above with laboratory-grown $\mathrm{MgO}$ crystals and minerals like olivine it was already known that the charge carriers which we expected to see in rocks would be positive holes that exist in an electrical inactive, dormant state, as positive hole pairs, PHPs, chemically equivalent to peroxy links, $\mathrm{O}_{3} \mathrm{X} / \mathrm{OO} \backslash \mathrm{XO}_{3}$. As defect electrons in the $\mathrm{O} 2 \mathrm{p}$-dominated valence band of the silicate minerals, chemically $\mathrm{O}^{-}$in an $\mathrm{O}^{2-}$ matrix, such positive holes should be capable of propagating through rocks over macroscopic distances with little apparent scatter or attenuation at grain boundaries.

Most relevant to earthquake-related phenomena is the observation that the PHPs can indeed be activated by steel balls impacting at a relatively low velocity and depositing $0.06 \mathrm{~J}$ or less in kinetic energy. The electrical, EM, and luminous signals produced suggest that the positive hole charge carriers propagate in a specific manner through the rocks. Examples are given in Figure la and Figure 2. The threshold for the generation of these charge carriers seems to be so low that the crackle of microfracturing in a rock volume under increasing stress is likely to have the same effect. Therefore, as microfractures open and close in rapid succession throughout such a rock volume, they emit acoustic 
wavelets. Each microfracture would generate positive holes, locally on a small scale, but in sum they would add up to a large charge cloud.

Molchanov and Hayakawa (1998a/b) introduce the concept of "electrification" by microfracturing as a possible mechanism for low-frequency EM emissions before earthquakes. The experiments described here provide a physical basis for such an "electrification". If microfractures open and close in different parts of the rock volume in rapid succession, each generating a cloud of positive holes, fluctuations in the charge carrier density are expected to produce current pulses, possibly propagating in different directions, generating a wideband EM noise and discrete pulsations as observed during the December 7, 1988, $\mathrm{M}_{\mathrm{s}}=7$ Spitak, Armenia, earthquake (Kopytenko et al., 1993).

With the knowledge now available that positive holes may be generated in rocks we can go one step further and address the phenomenon of earthquake lights. Reported in folklore (Tributsch, 1983), many times in the scientific literature (Derr, 1973), and photographed during the 1966 Matushiro earthquake, Japan (Derr, 1986), earthquake lights have remained a contentious issue. The reason is that no mechanism was known that could reasonably explain electric discharges at the rock-to-air interface and the appearance of "St. Elmo's fire." Neither piezoelectricity from quartz-bearing rocks (Finkelstein et al., 1973) nor sonoluminescence (Johnston, 1991) nor a fluid-driven dilatancy model (Nur, 1974) nor spray electrification by water that explosively evaporate out of the faults during a large magnitude earthquake (Lockner et al., 1983) seem to provide physical explanations for these luminous phenomena.

When positive hole clouds generated in the crust expand and reach the surface-to-air interface with its dielectric contrast, theory predicts (King and Freund, 1984) and the observations reported here confirm the appearance of surface potentials in the range of a few hundred $\mathrm{mV}$. Since the charge layer built from within the rock volume is very thin, of the order of a few hundred Angstrøm, the associated electric fields will be very high. For a charge carrier density of $10 \mathrm{ppm}\left(10^{17} \mathrm{~cm}^{-3}\right)$ the thickness of the charge layer at an interface with a dielectric contrast of 10 is $1000 \AA$ with a surface potential of the order of $400 \mathrm{mV}$ (King and Freund, 1984). This translates into an electric field across the flat surface of the order of $400,000 \mathrm{~V} \mathrm{~cm}^{-1}$. At rims and edges the electric field will be even higher and enough to cause dielectric breakdown of the air, corona discharges, light emission, and attendant bursts of EM radiation.

The photographs of earthquake lights during the 1966 earthquake swarm at Matsushiro, Japan, show the hilltops illuminated (Derr, 1986). This is consistent with an electric discharge where the electric fields are highest. In view of what we have learned through the impact experiments described here, it appears plausible that the charge carriers responsible for the earthquake lights during the 1966 Matsushiro event and on other occasions might have been positive holes, activated by the seismic waves.

A number of open questions remain. For instance, we have no quantitative data yet on the magnitude of the currents generated by the impacts nor the density of the positive holes activated at a given set of experimental parameters. Preliminary data on the concentration of PHPs suggest that in a typical igneous rock such as the Barre granite as much as $0.1-1 \%$ of all oxygen anions may be oxidized to the $\mathrm{O}^{-}$state. This means that, if all PHPs were activated, the positive hole density would reach values as high as $10^{19}-10^{20} \mathrm{~cm}^{-3}$. Theoretically (King and Freund, 1984) charge carrier densities of the order of $10^{17} \mathrm{~cm}^{-3}$ are enough to cause flat surface electric fields of the order of $400,000 \mathrm{~V} \mathrm{~cm}$, suggesting that activation of $1 / 100$ to $1 / 1000$ of the available PHPs would suffice to cause corona discharges at the rock surface.

We also don't know yet what the effect of water would be on the magnitude of the reported phenomena. However, having demonstrated that positive hole charge carriers exist in common igneous rocks opens a door to a new interpretation of electric, electromagnetic and luminous phenomena linked 
to earthquake activity, regardless whether water attenuates them as they propagate through a rock, leaves them unaffected or maybe even facilitates their passage.

\section{Acknowledgments.}

This work was performed without formal funding support. My thanks go to Jerome G. Borucki (NASA Ames Research Center) for his early involvement in sample preparation and data acquisition, to Michael Brunetti (California Monument, Inc.) and Michael J. Causi (Cañada Marble and Granite) for cutting rocks, to Bruce W. Fouke (University of Illinois at Urbana-Champaign) for petrographic analyses, and J. Thomas Dickinson (Washington State University) for constructive criticism at a crucial time of the experiments. Peter H. Schultz (Brown University) generously provided access to the NASA AVGR. Kraig Moll (Tektronix Instruments) made the 4-channel $500 \mathrm{MHz}$ oscilloscope available for the week of medium velocity impact experiments. Scott Hubbard and Douglas O'Handley (both NASA Ames Research Center) provided help through the NASA Ames Astrobiology Academy. Alexander S. Bradley (Harvard University and participant in the Astrobiology Academy 1998 Summer program) contributed to the data analysis. As reviewer, Stephen Park (University of California, Riverside) provided constructive criticism that helped improve the manuscript.

\section{References}

Bernabé, Y., Streaming potential in heterogenous networks, 1998. J. Geophys. Res., 103, 20,82720,841 .

Bernard, P., Plausiblity of long distance electrotelluric precursors of earthquakes, 1992. J. Geophys. Res., 97, 17,531-17,546.

Bilichenko, S.V., F.S. Iljin, and E.F. Kim, ULF response of the ionosphere for earthquake preparation processes, 1990. Dokl. Acad. Nauk USSR, 311, 1077-1080.

Bishop, J.R., 1981. Piezoelectric effects in quartz-rich rocks, Tectonophys., 77, 297-321.

Bolt, B.A., 1988. Earthquakes, W. H. Freeman and Company, New York.

Brady, B.T., and G.A. Rowell, 1986. Laboratory investigation of the electrodynamics of rock fracture, Nature, 321, 488-492.

Chmyrev, V.M., V.N. Isaev, S.V. Bilichenko, E.P. Trushkina, and G. Stanev, 1986. Electric fields and hydromagnetic waves in the ionosphere above an earthquake center, Geomagnetizm i Aeronomiia, 26, 1020-1022.

Christensen, N.I., 1982. Seismic velocities, in Handbook of Physical Properties of Rocks, pp. 1-228, CRC', Baton Rouge, FL.

Constable, S., and A. Duba, 1990. Electrical conductivity of olivine, a dunite and the mantle, J. Geophys. Res., 95, 6967-6978.

Crawford, D.A., and P.H. Schultz, 1991. Laboratory investigations of impact-generated plasma, J. Geophys. Res., 96, 18,807-18,817.

Davis, K., and D.M. Baker, Ionospheric effects observed around the time of the Alaskan earthquake of March 28.1964, 1965. J. Geophys. Res., 70, 2251-2253.

Derr, J.S., 1973. Earthquake lights: a review of observations and present theories, Bull. Seismol. Soc. Amer., 63, 2177-21287.

Derr, J.S., 1986. Luminous phenomena and their relationship to rock fracture, Nature, 321, 470-471.

Dickinson, J.T., L.C. Jensen, M.R. McKay, and F. Freund, 1986. The emission of atoms and molecules accompanying fracture of single-crystal magnesium oxide, J. Vac. Sci. Technol., 4, 1648-1652.

Dobrovolsky, I.P., N.I. Gershenson, and M.B. Gokhberg, 1989. Theory of electrokinetic effects occurring at the final stage in the preparation of a tectonic earthquake, Phys. Earth Planet. Int., 57, 144-156. 
Draganov, A.B., U.S. Inan, and Y.N. Taranenko, 1991. ULF magnetic signatures at the Earth surface due to ground water flow: a possible precursor to earthquakes, Geophys. Res. Lett., 18, 1127 1130.

Drobgev, V.I., V.M. Krasnov, and R.M. Salihov, 1978. About ionospheric perturbation associated with earthquake and explosions, Izv. Vyssh. Uchebn. Zaved., 21, 1862-1978.

Edwards, A.H., and W.B. Fowler, 1982. Theory of the peroxy-radical defect in a-SiO 2 . Phys. Rev. B, 26, 6649-6660.

Eiby, G.A., 1980. Earthquakes, Van Nostrand Reinhold Company, New York, NY.

Emmermann, R., and J. Lauterjung, 1997. The German continental deep drilling program KTP: Overview and major results, J. Geophys. Res., 102, 18,179-18,201.

Enomoto, Y., and H. Hashimoto, 1990. Emission of charged particles form indentation fracture of rocks, Nature, 346, 641-643.

Finkelstein, D., U.S. Hill, and J.R. Powell, 1973. The piezoelectric theory of earthquake lightning, J. Geophys. Res., 78, 992-993.

Fraser-Smith, A.C., A. Bernardi, P.R. McGill, M.E. Ladd, R.A. Helliwell, and O.G. Villard, 1990. Low-frequency magnetic field measurements near the epicenter of the $\mathrm{Ms}=7.1$ Loma Prieta earthquake, Geophys. Res. Lett., 17, 1465-1468.

Freund, F., 2000. Time-resolved study of charge generation and propagation in igneous rocks J. Geophys. Res. 105, 11,001-11,019.

Freund, F., and J.G. Borucki, 1999. Charge carrier generation and charge cloud propagation following $100 \mathrm{~m} / \mathrm{s}$ impacts on igneous rocks, in Atmospheric and Ionospheric Electromagnetic Phenomena Associated with Earthquakes, edited by M. Hayakawa, pp. 839-857, Terra Sci. Publ., Tokyo.

Freund, F., M.M. Freund, and F. Batllo, 1993. Critical review of electrical conductivity measurements and charge distribution analysis of MgO, J. Geophys. Res., 98, 22,209-22,229.

Freund, F., and M.M. Masuda, 1991. Highly mobile oxygen hole-type charge carriers in fused silica, J. Mater. Res., 8, 1619-1622.

Freund, F., and H. Wengeler, 1982. The infrared spectrum of $\mathrm{OH}$--compensated defect sites in C-doped $\mathrm{MgO}$ and $\mathrm{CaO}$ single crystals, J. Phys. Chem. Solids, 43, 129-145.

Freund, F., Whang, E.-J., Batllo, F., Desgranges, L., Desgranges, C., and Freund, M.M., 1994. Positive hole-type charge carriers in oxide materials, in: Grain Boundaries and Interfacial Phenomena in Electronic Ceramics, edited by L.M. Levinson, pp. 263-278, Amer. Ceram. Soc. Cincinnati, OH Friebele, E.J., D.L. Griscom, M. Stapelbroek, and R.A. Weeks, 1979. Fundamental defect centers in glass: The peroxy radical in irradiated high-purity fused silica, Phys. Rev. Lett., 42, 1346-1349.

Fujinawa, Y. and K. Takahashi, 1990. Emission of electromagnetic radiation preceding the Ito seismic swarm of 1989 , Nature, 347, 376-378.

Galperin, Y.I., G. V.A., N.V. Jorjro, R.A. Kavrazhkin, Y.V. Lissakov, V.D. Maslov, L.M. Nikolaenko, R.Z. Saddeev, O.A. Molchanov, M.M. Mogilevsky, L.S. Alperovich, M.B. Gokhberg, E.A.

Ivanov, O.A. Pokhotelov, H. Reme, J.M. Bosqued, C. Beghin, and J.J. Berthelier, 1985. VLF and ELF effects in the upper ionospheric caused by large-scaled acoustic waves in the lower ionosphere observed from aurol-3 satellite, Result of the ARCAD-3 Project, Cepadues, Toulouse, 661-684.

Geller, R.J., 1996. Debate on VAN, Geophys. Res. Lett., 23 (whole issue \#11).

Geller, R.J., 1997. Earthquake prediction: a critical review, Geophys. J. Int., 131, 425-450.

Hadjicontis, V., and C. Mavromatou, 1994. Transient electric signals prior to rock failure under uniaxial stress, Geophys. Res. Lett., 21, 1687-1690.

Hedervari, P., and Z. Noszticzius, 1985. Recent results concerning earthquake lights, 3, 705-708.

Horn, R.G., and D.T. Smith, 1992. Contact electrification and adhesion between dissimilar materials,

Science, 256, 362-365. 
Huang, Q., and M. Ikeya, 1998. Seismic electromagnetic signals (SEMS) explained by a simulation experiment using electromagnetic waves, Phys. Earth Planet. Inter., 109, 107-114.

Johnston, A.C., 1991. Light from seismic waves, Nature, 354, 361.

Johnston, M.J.S., 1997. Review of electric and magnetic fields accompanying seismic and volcanic activity, Surveys in Geophysics, 18, 441-475.

Johnston, M.J.S., and M. Parrot, 1989. Seismoelectromagnetic Effects, Phys. Earth Planet. Inter., (Special Issue), 57, 1-177.

King, B.V., and F. Freund, 1984. Surface charges and subsurface space charge distribution in $\mathrm{MgO}$ containing dissolved traces of water. Phys. Rev., B 29, 5814-5824.

Kopytenko, Y.A., T. Matiashvili, P.M. Voronov, E.A. Kopytenko, and O.A. Molchanov, 1993. Detection of ultra-low-frequency emissions connected with the Spitak earthquake and its aftershock activity, based on geomagnetic pulsation data at Dusheti and Vardzia observatories, Phys. Earth Planet. Inter., 77, 88-95.

Kustov, V.A., and Liperovsky, 1988. About perturbations in the ionospheric F-region before strong earthquakes, Izv. Akad. Nauk USSR, Fiz. Zemli, N4, 12-20.

Larkina, V.I., V.V. Migulin, O.A. Molchanov, A.S. Ilchin, and V.V. Shvetsova, 1988. Characteristics of the excitation of low-frequency emissions in the upper ionosphere above earthquake regions, Geomagn. Aeron., 28, 685-689.

Lee, C. C., J. Y. Liu and C. J. Pan, 2000. The height of sporadic-E layer simultaneously observed by the VHF radar and ionosondes in Chung-Li. Geophys. Res. Lett. 27: 641-644.

Liu, J. Y., Y. H. Chu, M. Q. Chen, L. C. Tsai and C. M. Huang, 2000. Modeling and ground observations of the ionosphere related to the COSMIC project. Terr. Atmo. Ocean. Sci. 11: 349364.

Loeb, I.B., 1958. Static Electrification, Springer, Berlin.

Lockner, D.A., Johnston, M.J.S., and Byerlee, J.D., 1983. A mechanism to explain the generation of earthquake lights. Nature 302, 28-32.

Martens, R., H. Gentsch, and F. Freund, 1976. Hydrogen release during the thermal decomposition of $\mathrm{Mg}(\mathrm{OH})_{2}$ to $\mathrm{MgO}$, J. Catalysis, $44,366-372$.

Masood, E., 1995. Greek earthquake stirs controversy over claims for prediction, Nature, 375, 617.

Merzer, M., and S.L. Klemperer, 1997. Modeling low-frequency magnetic-field precursors to the Loma Prieta earthquake with a precursory increase in fault-zone conductivity, Pure Appl. Geophys., 150, 217-248.

Meyer, L.L., 1977. California Quake, Sherbourne Press, Nashville, TN.

Michael, A.J., 1996. Testing prediction methods: Earthquake clustering versus the Poisson model, Geophys. Res. Lett., 24, 1891-1894.

Molchanov, O.A., and M. Hayakawa, 1998a. Subionospheric VLF signal perturbations possibly related to earthquakes, J. Geophys. Res., 103 (A8), 17,489-17,504.

Molchanov, O.A., and M. Hayakawa, 1998b. On the generation mechanism of ULF seismogenic electromagnetic emissions, Phys. Earth Planet. Inter., 105, 201-210.

Molchanov, O.A., M. Hay akawa, T. Oudoh, and E. Kawai, 1998. Precursory effects in the subionospheric VLF signals for the Kobe earthquake, Phys. Earth Planet. Interiors, 105, 239-248.

Molchanov, O.A., Y.A. Kopytenko, E.A. Kopytenko, T. Matiashvili, A.C. Fraser-Smith, and A. Bernardi, 1992. Results of ULF magnetic field measurements near the epicenters of the Spitak $(\mathrm{Ms}=6.9)$ and Loma Prieta $(\mathrm{Ms}=7.1)$ earthquakes: Comparative analysis, Geophys. Res. Lett., 19, 1495-1498.

Morat, P., and J.L. Le Mouel, 1987. Variation of the electric resistivity of large rock samples with stress, Geophysics, 52, $1424-1430$. 
Morat, P., and J.-L. LeMouël, 1992. Electrical signals generated by stress variations in porous nonsaturated rocks, C.R. Acad. Sci. Ser. II, 315, 955-963.

Morrison, F.D., E.R. Williams, and T.D. Madden, 1989. Streaming potentials of Westerly granite with applications, J. Geophys. Res., 94, 12449-12461.

Nur, A., 1974. Matsushiro, Japan, earthquake swarm: Confirmation of the dilatancy-fluid diffusion model, Geology, 2, 217-221.

Oommen, T.V., 1988. Static electrification properties of transformer oil, IEEE Transaction on Electrical Insulation, 23, 123-128.

Park, S.K., 1997a. Electromagnetic precursors to earthquakes: a search for predictors, Science Progress, 80, 65-82.

Park, S.K., 1997b. Monitoring resistivity change in Parkfield, California: 1988-1995, J. Geophys. Res., $102,24,545-24,559$.

Park, S.K., M.J.S. Johnston, T.R. Madden, F.D. Morgan, and H.F. Morrison, 1993. Electromagnetic precursors to earthquakes in the ULF band: a review of observations and mechanisms, Reviews Geophys., 31, 117-132.

Pham, V.N., D. Boyer, G. Chouliaras, J.L. Le Mouel, J.C. Rossignol, and G.N. Stavrakakis, 1998. Characteristics of electromagnetic noises in Ioannina region (Greece): a possible origin for so called "Seismic Electric Signals" (SES), Geophys. Res. Lett., 25, 2229-2232.

Quellet, M., 1990. Earthquake light and seismicity, Nature, 348, 492.

Rikitake, T., 1976. Earthquake Prediction, Elsevier Sci. Publ. Co., Amsterdam.

Rossman, G.R., 1996. Studies of $\mathrm{OH}$ in nominally anhydrous minerals, Phys. Chem. Minerals, 23, 299-304.

Schock, R.N., and A. Duba, 1985. Point defects and the mechanisms of electrical conduction in olivine, in Point Defects in Minerals, edited by R.N. Schock, pp. 88-96, Amer. Geophys. Union, Washington, D.C.

Serebryakova, O.N., S.V. Bilichenko, V.M. Chmyrev, M. Parrot, J.L. Rauch, F. Lefeuvre, and O.A. Pokhotelov, 1992. Electromagnetic ELF radiation from earthquake regions as observed by lowaltitude satellites, Geophys. Res. Lett., 19, 91-94.

Shankland, T.J., 1981. Electrical conduction in mantle materials., in Evolution of the earth, edited by R.J. O'Connell, and W.S. Fyfe, pp. 256-263, Amer. Geophys. Union, Washington, D.C.

Shankland, T.J., and M.E. Ander, 1983. Electrical conductivity, temperature and fluids in the crust, J. Geophys. Res., 88, 9475-9484.

Tributsch, H., 1983. When Snakes Awake: Animals and Earthquake Prediction, MIT Press, Cambridge, Mass.

Varotsos, P., N. Sarlis, M. Lazaridou, and P. Kapiris, 1997. Transmission of stress induced electric signals in dielectric media, J. Appl. Phys., 83, 60-70.

Yoshida, S., O.C. Clint, and P.R. Sammonds, 1998. Electric potential changes prior to shear fracture in dry and saturated rocks, Geophys. Res. Lett., 25, 1577-1580.

Yoshida, S., M. Uyeshima, and M. Nakatani, 1997. Electric potential changes associated with slip failure of granite: Preseismic and coseismic signals, J. Geophys. Res., 102, 14,883-14,897.

Zhijia, Z., 1989. Investigation of tectonomagnetic phenomena in China, Phys. Earth Planet. Int., 57, 1122. 


\section{Figures}
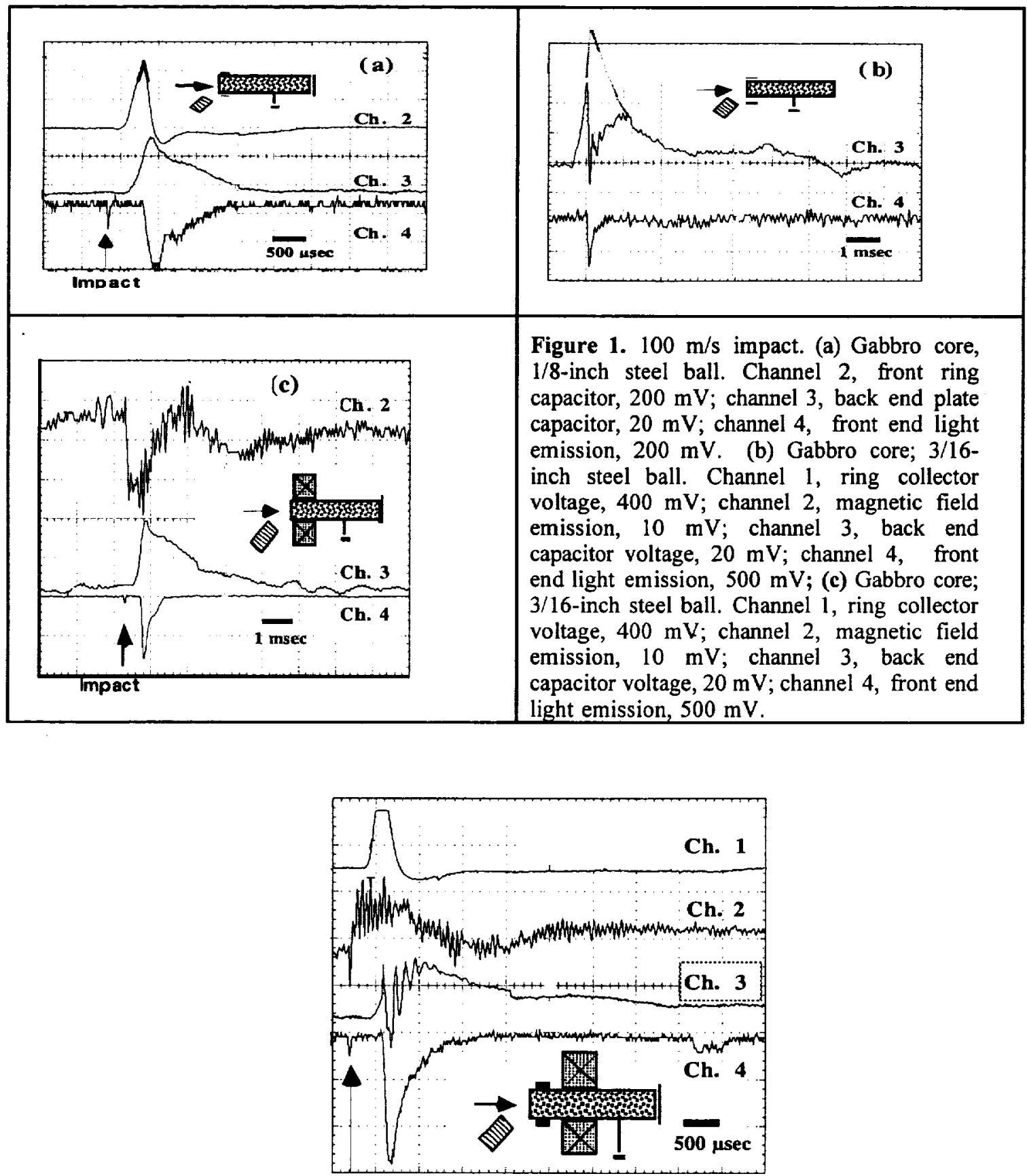

Impact

Figure 2. A $100 \mathrm{~m} / \mathrm{s}$ impact. Diorite core; 3/16-inch steel ball. Channel 1 , ring electrode voltage, $400 \mathrm{mV}$; channel 2, EM emission, $10 \mathrm{mV}$; channel 3, back end capacitor voltage, $20 \mathrm{mV}$; channel 4, front end light emission, $500 \mathrm{mV}$. 

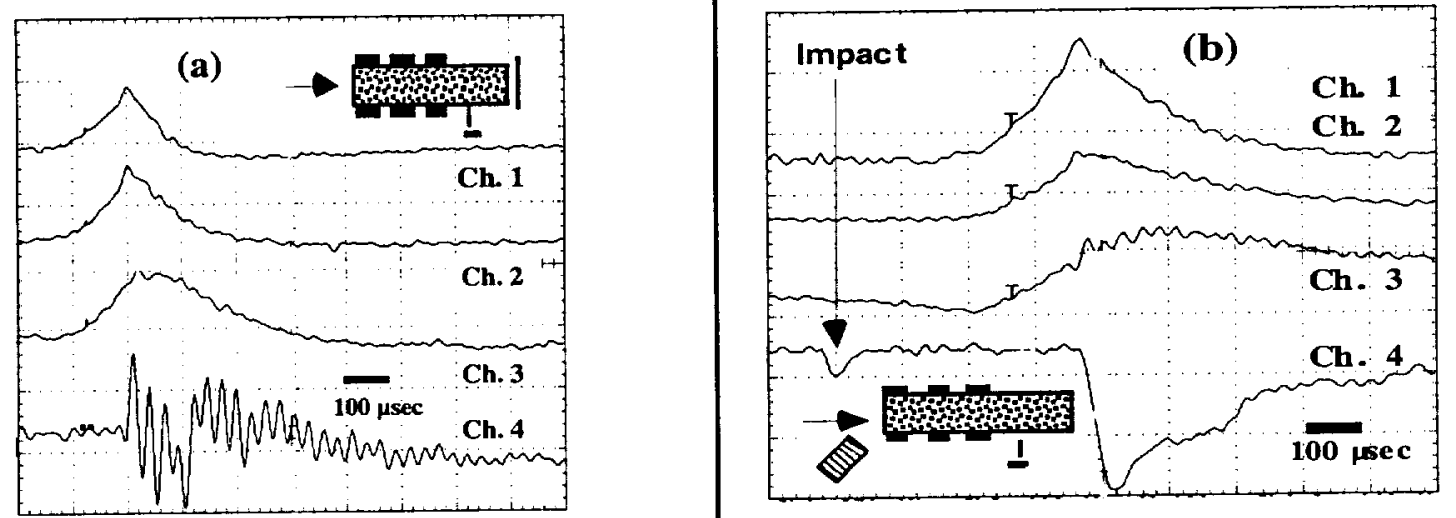

Figure 3. A $100 \mathrm{~m} / \mathrm{s}$ impact. Diorite, 3/16-inch steel balls. (a) Channels 1-3, three ring collector electrodes, $500 \mathrm{mV}$, $100 \mathrm{mV}$ and $20 \mathrm{mV}$, respectively; channel 4, back end capacitor voltage, $5 \mathrm{mV}$. Note the kilohertz oscillations of the back end capacitor voltage and the $38-\mathrm{kHz}$ ripple on the three ring collectors after the onset of the light emission; (b) Channels $1-3$, three ring collector electrodes, $500 \mathrm{mV}, 100 \mathrm{mV}$ and $20 \mathrm{mV}$, respectively; channel 4 , front end light emission, $1 \mathrm{~V}$.

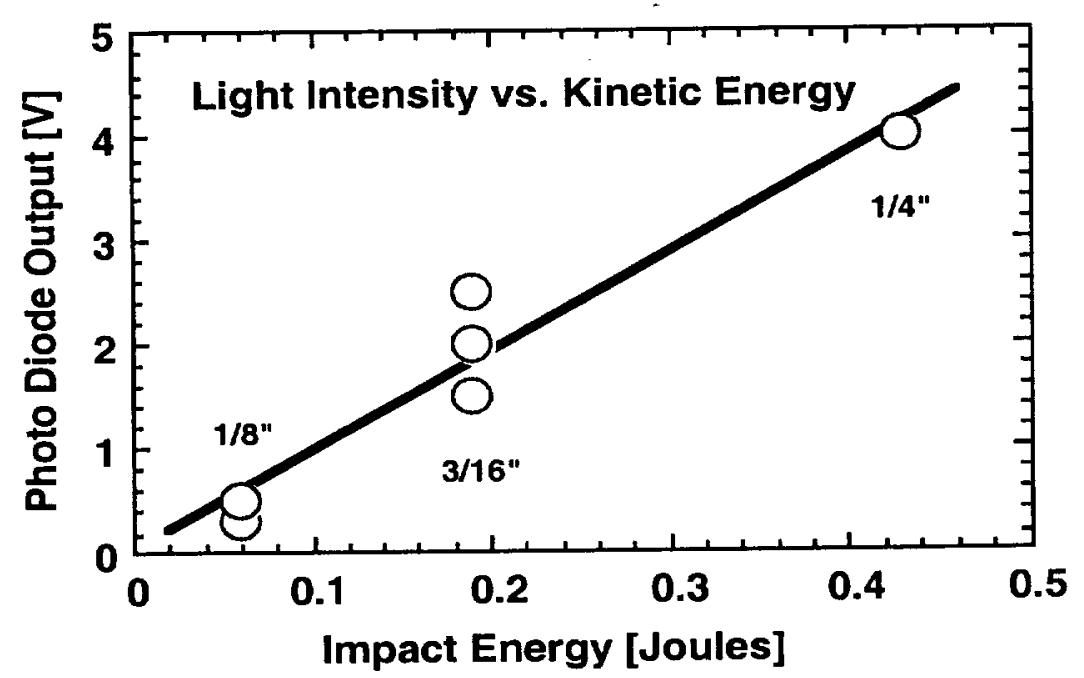

Figure 4: Probable linear relation between the integrated intensity of the delayed light emitted from the rock cores and the kinetic energy of impact. 


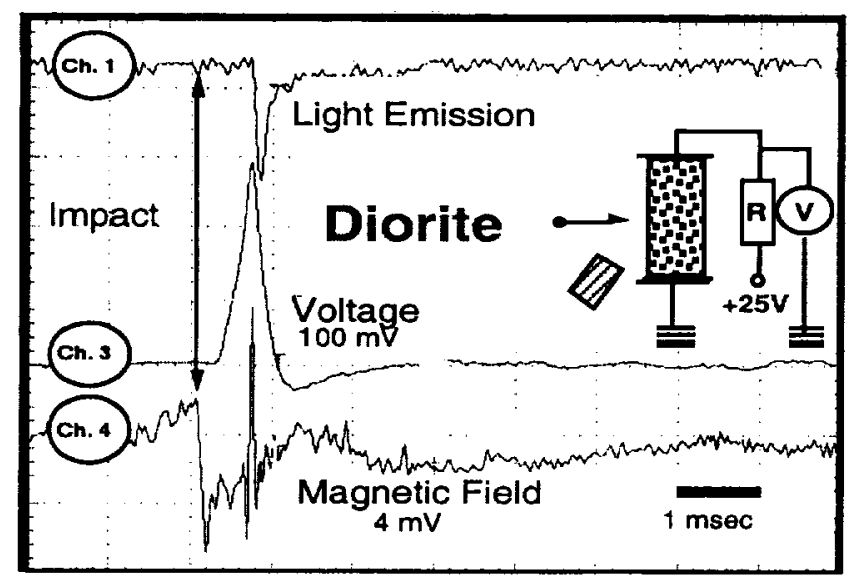

Figure 5. A $100 \mathrm{~m} / \mathrm{s}$ impact. Cross current measurement with a rectangular piece of diorite impacted at right angle. Channel 1, light emission; channel 3, cross current; channel 4, EM emission (magnetic pick-up coil not shown in inset).

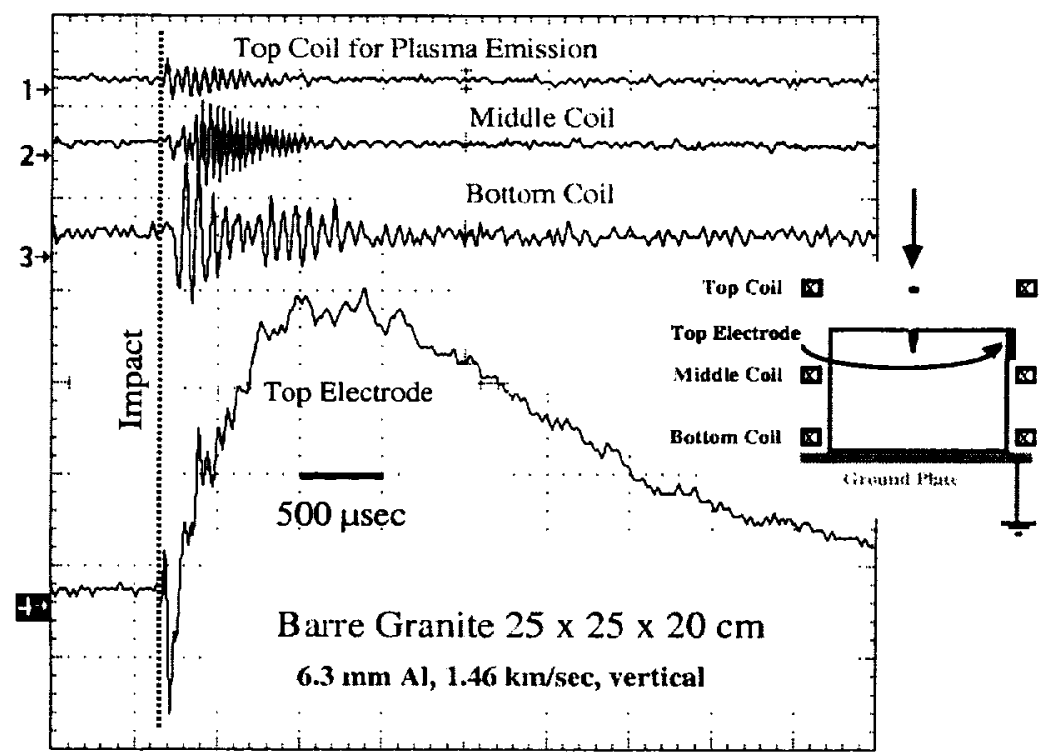

Figure 6. The $1.46 \mathrm{~km} / \mathrm{s}$ impact of shot 4 on granite with the signals from the three magnetic pick-up coils and the top contact electrode positioned as shown in inset, using the $200-\mathrm{MHz}$ oscilloscope model 420. 

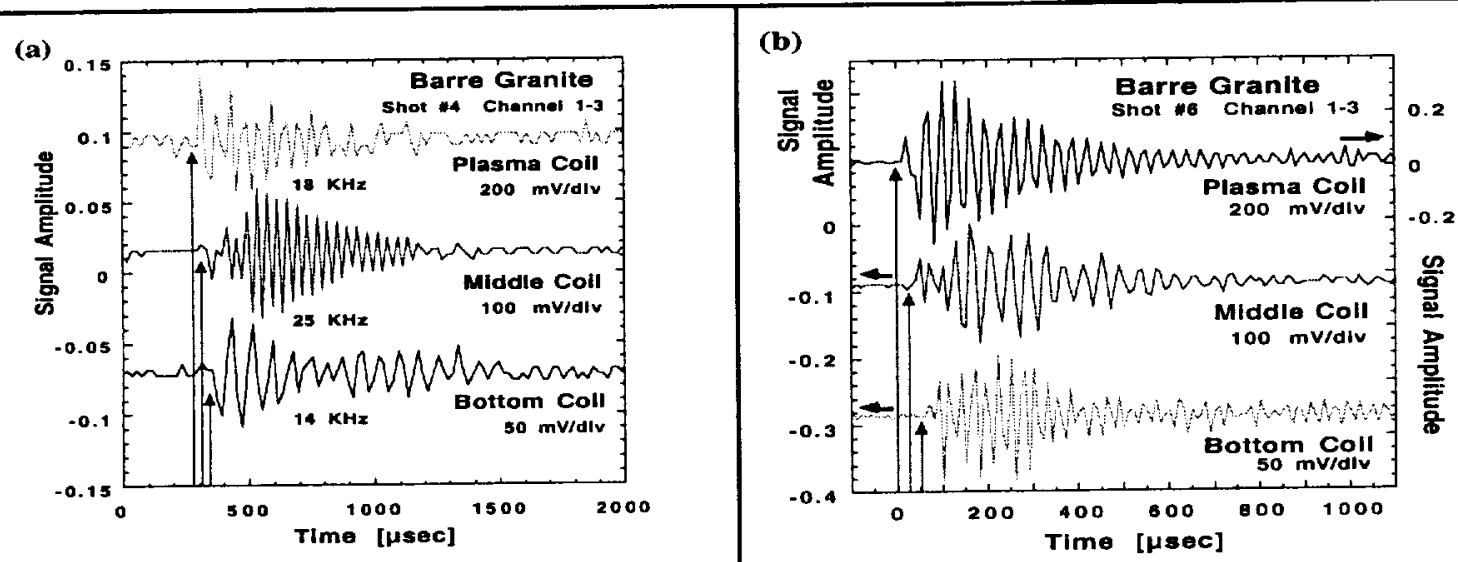

Figure 7. Electromagnetic emission as recorded by the magnetic pick-up coils after impact of the Al sphere (a) during shot 4 at $1.46 \mathrm{~km} / \mathrm{s}$, (b) during shot 6 at $4.45 \mathrm{~km} / \mathrm{s}$ and (c) during shot 7 at $5.64 \mathrm{~km} / \mathrm{s}$ which led to disintegration of the granite block.
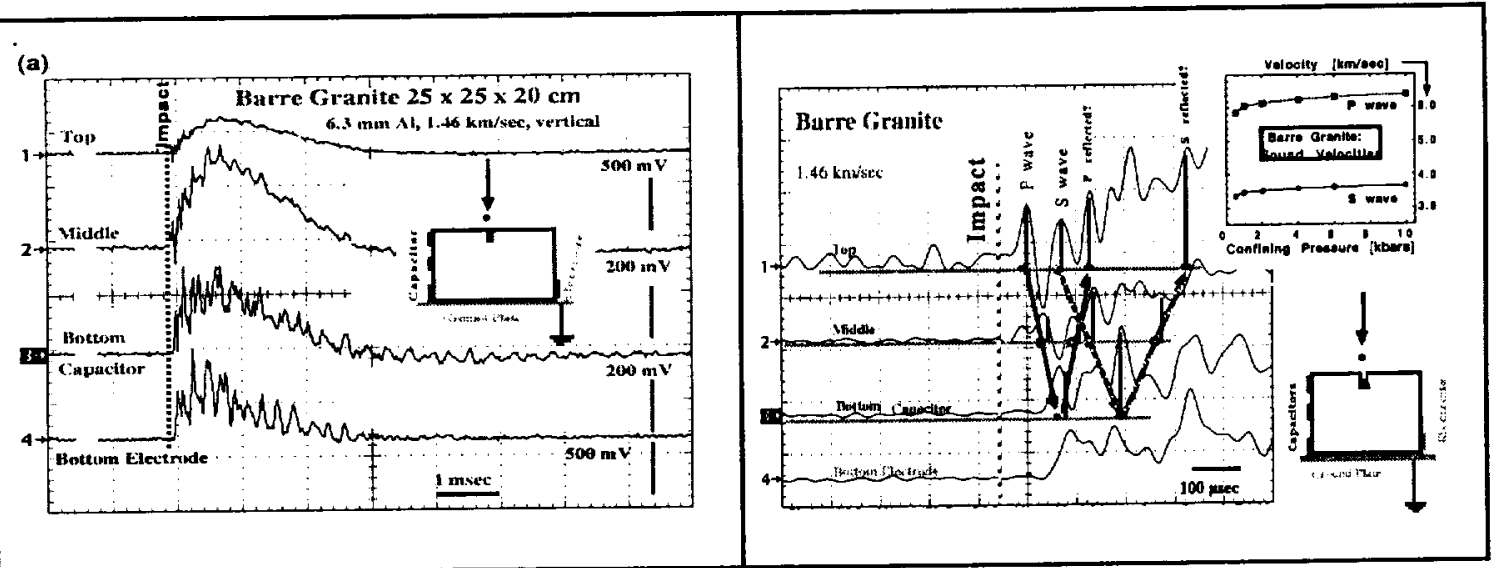

Figure 8. The $1.46 \mathrm{~km} / \mathrm{s}$ impact of shot 4 on the granite block. (a) Signals from the three capacitive sensors and the bottom contact electrode positioned as shown in the inset, using the $500-\mathrm{MHz}$ oscilloscope model 754 . (b) Initial arrival of the signals at higher time resolution with tentative assignment of the short voltage pulses to piezoelectric signals arising from the passing of the incoming and reflected(?) $\mathbf{P}$ and $\mathbf{S}$ waves. Inset shows velocities of the $\mathbf{P}$ and $\mathbf{S}$ waves in Barre Granite as a function of the confining pressure after Christensen (1982). 


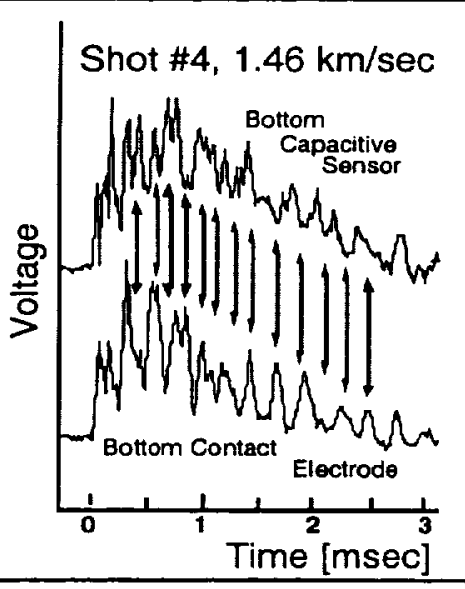

Figure 9.

Out-of-phase correlation between the voltage signal as recorded by the bottom capacitive sensor (top trace) and the electron injection into the rock as recorded by the bottom contact electrode (bottom trace).

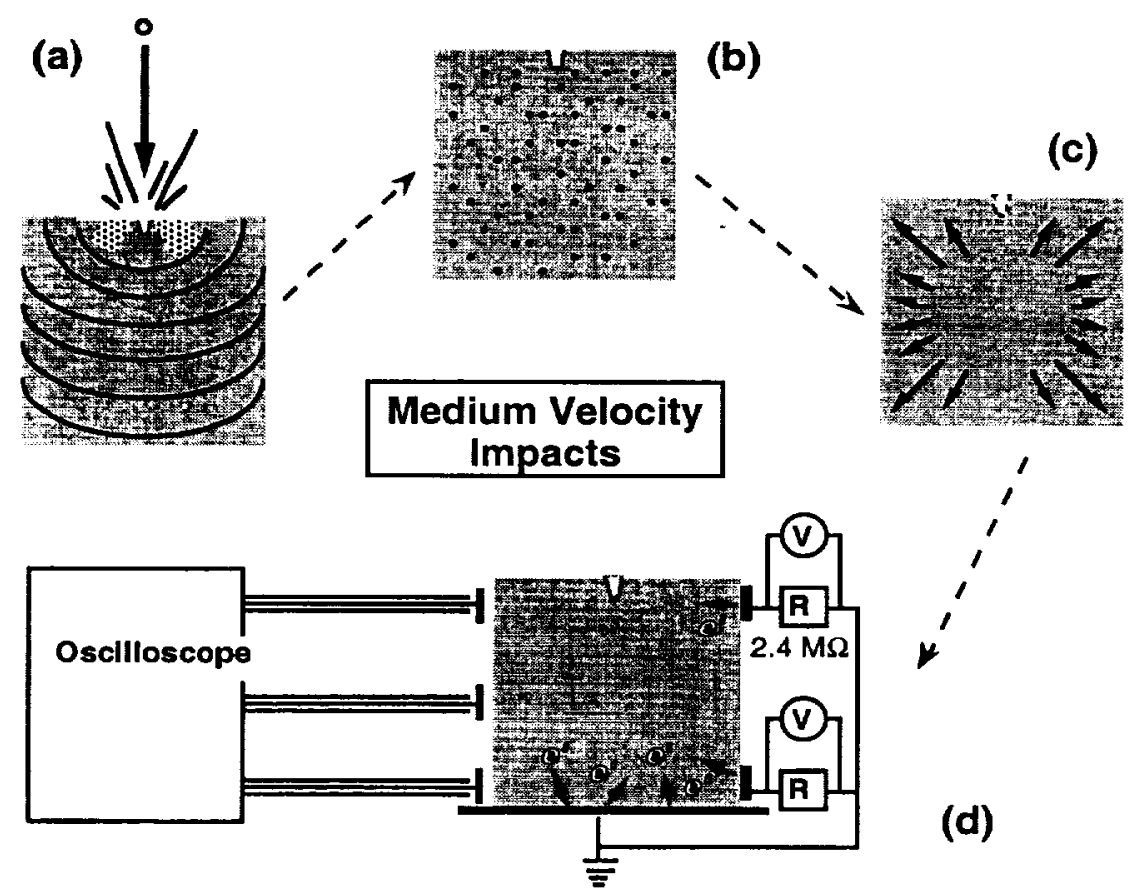

Figure 10. Cartoon of the processes taking place in rapid succession in the granite block following a medium velocity impact. (a) Impact and propagation of the sound waves. (b) Rock volume filled with positive hole charge carriers activated in the wake of the sound waves. (c) Diffusion of positive holes to the surface due to mutual repulsion in the bulk. (d) Injection of electrons from the base plate and contact electrodes leading to oscillations. 


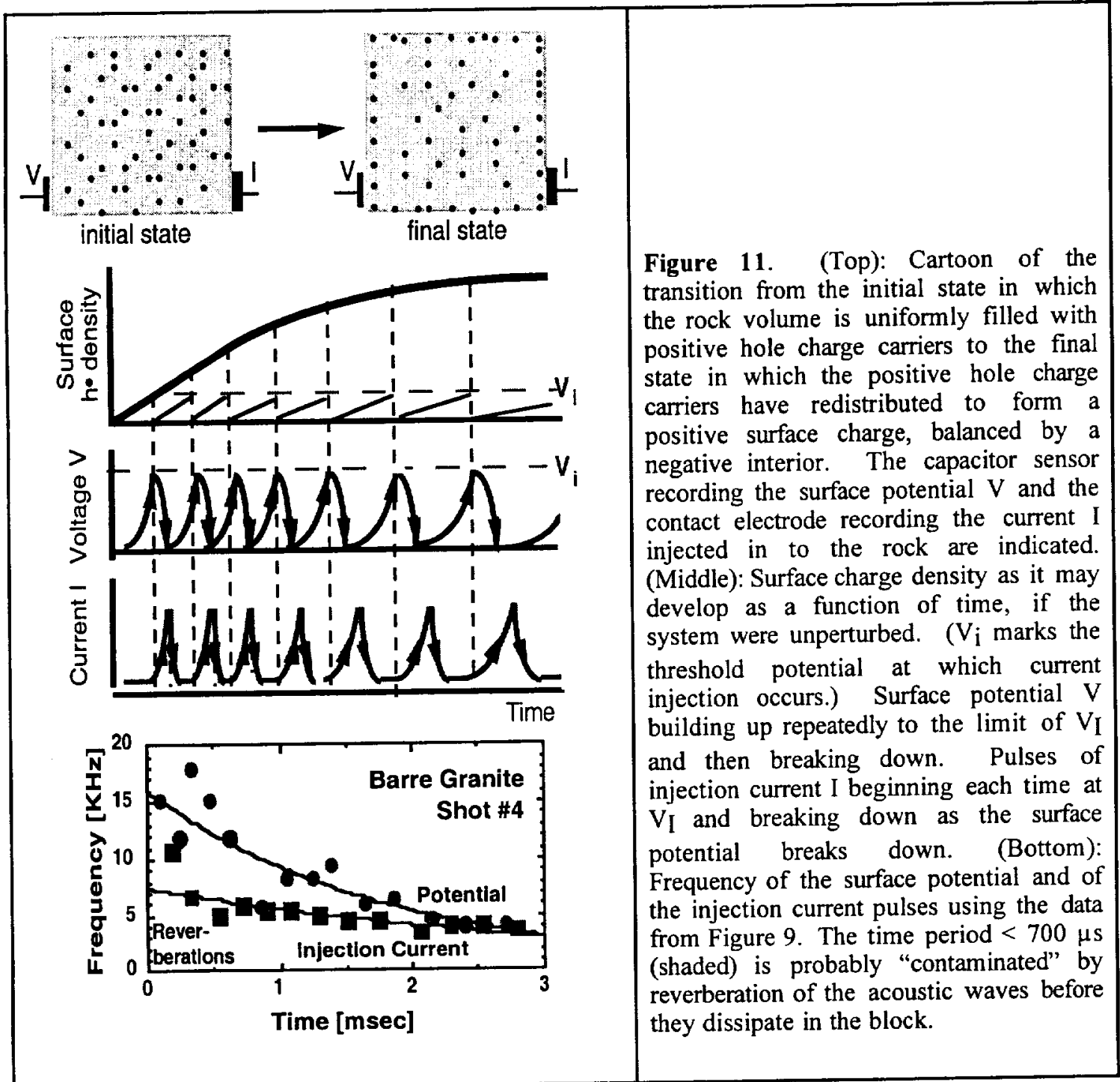


—ב_ 